MATHEMATICS OF COMPUTATION

Volume 79, Number 272, October 2010, Pages 2033-2058

S $0025-5718(2010) 02384-1$

Article electronically published on May 24, 2010

\title{
MULTIGRID IN A WEIGHTED SPACE ARISING FROM AXISYMMETRIC ELECTROMAGNETICS
}

\author{
DYLAN M. COPELAND, JAYADEEP GOPALAKRISHNAN, AND MINAH OH
}

\begin{abstract}
Consider the space of two-dimensional vector functions whose components and curl are square integrable with respect to the degenerate weight given by the radial variable. This space arises naturally when modeling electromagnetic problems under axial symmetry and performing a dimension reduction via cylindrical coordinates. We prove that if the original threedimensional domain is convex, then the multigrid $\mathrm{V}$-cycle applied to the inner product in this space converges, provided certain modern smoothers are used. For the convergence analysis, we first prove several intermediate results, e.g., the approximation properties of a commuting projector in weighted norms, and a superconvergence estimate for a dual mixed method in weighted spaces. The uniformity of the multigrid convergence rate with respect to mesh size is then established theoretically and illustrated through numerical experiments.
\end{abstract}

\section{INTRODUCTION}

Multigrid methods can be made to work for problems involving the curl and divergence operators, notwithstanding their large kernels, as is now well known, thanks to the papers 2, 22, The purpose of this work is to extend such techniques to axisymmetric problems where one only has control of curl through a weighted norm. Weighted Sobolev spaces arise naturally when deriving the weak formulations of standard boundary value problems under axial symmetry. Using cylindrical coordinates $(r, \theta, z)$, we can reduce three-dimensional (3D) problems in unweighted spaces to two-dimensional (2D) ones set in weighted spaces, the weight function being the radial coordinate $r$. The dimensional reduction from 3D to $2 \mathrm{D}$ is an attractive feature as it can significantly reduce the computational expense. But when the axis of symmetry is contained in the domain, the degeneracy of the weight $r$ necessitates careful mathematical treatment. Our concern in this paper is the rigorous study of the convergence of the multigrid applied to a bilinear form in one such weighted space, namely $\boldsymbol{H}_{r}(\operatorname{curl}, D)$, which we now define.

It is a space of vector functions $\boldsymbol{v}$ on the $(r z)$-plane with certain integrability properties. Letting the components of $\boldsymbol{v}$ be denoted by $v_{r}$ and $v_{z}$, and the curl by

$$
\operatorname{curl}_{r z} \boldsymbol{v}=\partial_{z} v_{r}-\partial_{r} v_{z},
$$

Received by the editor November 17, 2008 and, in revised form, June 11, 2009.

2010 Mathematics Subject Classification. 65M55, 65N55, 65F10, 65N30, 78M10.

Key words and phrases. Multigrid, axisymmetric, weighted Sobolev spaces, Maxwell equations, V-cycle, duality, superconvergence, mixed method, finite element.

This work was supported in part by the National Science Foundation under grants DMS0713833 and SCREMS-0619080. 
we define

$$
\boldsymbol{H}_{r}(\operatorname{curl}, D)=\left\{\boldsymbol{v} \in L_{r}^{2}(D)^{2}: \operatorname{curl}_{r z} \boldsymbol{v} \in L_{r}^{2}(D)\right\} .
$$

Here $D$ is a polygonal subset of the $(r z)$-plane and $L_{r}^{2}(D)$ is the set of all real-valued measurable functions on $D$ with $\int_{D} u^{2} r d r d z<\infty$. It is well known that $L_{r}^{2}(D)$ is a Hilbert space with the weighted inner product defined by

$$
(u, v)_{r}=\int_{D} r u v d r d z
$$

The space $\boldsymbol{H}_{r}(\operatorname{curl}, D)$ is also Hilbert with the inner product

$$
\Lambda(\boldsymbol{u}, \boldsymbol{v})=\left(\operatorname{curl}_{r z} \boldsymbol{u}, \operatorname{curl}_{r z} \boldsymbol{v}\right)_{r}+(\boldsymbol{u}, \boldsymbol{v})_{r} .
$$

The goal of this paper is to analyze the application of the multigrid to the bilinear form $\Lambda(\cdot, \cdot)$.

Bilinear forms such as $\Lambda(\cdot, \cdot)$ arise when deriving variational formulations from the Maxwell equations in cases when the material properties and geometry are axisymmetric. Since such details have been well explained by many authors [3, 8. 17, we shall not dwell on them here. It suffices to say that the 3D Maxwell system (consisting of six scalar equations for the six components of the electric and magnetic fields) decouples into two systems of three equations each, one called the azimuthal problem and the other the meridian problem. The meridian problem is for the components in the right half of the $(r z)$-plane (sometimes called the meridian half-plane) of the electric field, which we may denote by $\boldsymbol{E}_{r z}=E_{r} \boldsymbol{e}_{r}+E_{z} \boldsymbol{e}_{z}$. The components $E_{r}$ and $E_{z}$ are functions of $r$ and $z$ alone (as there is no $\theta$ dependence due to axial symmetry). In the time-harmonic case, $\boldsymbol{E}_{r z}$ satisfies

$$
\operatorname{curl}_{r z}\left(\frac{1}{\mu} \operatorname{curl}_{r z} \boldsymbol{E}_{r z}\right)-\omega^{2} \epsilon \boldsymbol{E}_{r z}=\boldsymbol{F},
$$

where $\mu$ is the magnetic permeability, $\epsilon$ is the dielectric constant, $\boldsymbol{F}$ represents given sources, all of which are axisymmetric, and $\omega$ is the wave number. Note that in (1.3), while $\operatorname{curl}_{r z}(\cdot)$ is as defined in (1.1), the operator $\operatorname{curl}_{r z}(\cdot)$ takes scalar functions to vector functions by

$$
\operatorname{curl}_{r z} \phi \equiv\left(-\partial_{z} \phi, \frac{1}{r} \partial_{r}(r \phi)\right) .
$$

Throughout this paper we say that a scalar-valued function is "axisymmetric" if it is is invariant under any rotation about the $r=0$ axis. A vector function is said to be axisymmetric if its scalar components in cylindrical coordinates are axisymmetric. The variational formulation for equations such as (1.3) are naturally posed in $\boldsymbol{H}_{r}(\operatorname{curl}, D)$.

Although the above-mentioned meridian problem gives rise to a complex and indefinite variational formulation in $\boldsymbol{H}_{r}(\operatorname{curl}, D)$, a first step towards analyzing multigrid algorithms for it would be an analysis of the multigrid algorithm applied to the $\boldsymbol{H}_{r}(\operatorname{curl}, D)$-inner product over the real field. Even with this simplification, the analysis is nontrivial because of the difficulties caused by the kernel of the curl operator and the degenerate weight. Techniques to overcome the former difficulty are now well known, thanks to the papers [1, 2, 22, and we shall make extensive use of their ideas. Some important results to handle difficulties caused by the degenerate weight are available in [17, 18, 21. However, we need to develop more tools in weighted spaces of vector-valued functions arising in our problem. 
Mathematical analysis of finite elements for axisymmetric problems is a subject of increasing interest. Many of the basic properties of the Sobolev spaces needed for scalar problems and Stokes flow were established in [6], while the same for the Maxwell system were proved more recently in 3. Simple finite elements for the azimuthal problem were analyzed in 21. This was followed by an analysis of finite elements for the meridian problem [17. Other studies on the performance of finite elements for axisymmetric Maxwell equations include [14, 18, 24. Previous papers have also investigated the application of multigrid techniques to the weighted spaces arising from axisymmetric Maxwell equations. Multigrid methods for the azimuthal problem are analyzed in [21. In [8] one finds another multigrid analysis, but using line relaxations. In contrast, our aim in this paper is the analysis of the V-cycle multigrid algorithm using the less expensive smoothers of [2] and [22]. This has a direct application to time-dependent and potential applications to time-harmonic meridian problems.

Since the applications we have in mind are those reduced from three to two dimensions by axial symmetry, we think of the domain $D$ as the restriction to the meridian half-plane of some $3 \mathrm{D}$ axisymmetric domain $\Omega$. In particular, we have in mind cases when the boundary $\partial D$ intersects the $r=0$ axis. We assume that $D$ is a bounded, connected domain with a Lipschitz polygonal boundary $\partial D$ partitioned into two disjoint subsets, namely its intersection with the $r=0$ axis, denoted by $\Gamma_{0}$, and the remainder, denoted by $\Gamma_{1}$. If $\Gamma_{0}$ is empty, the weight function $r$ is not degenerate and there is little difficulty in extending the known techniques. Here we consider the case when $\Gamma_{0}$ has a positive (one-dimensional) measure. Furthermore, to avoid topological complications, we assume that $\Gamma_{1}$ is connected and that $D$ is simply connected.

In addition to $\boldsymbol{H}_{r}(\operatorname{curl}, D)$, we shall also need a number of other weighted spaces. For the convenience of the reader, we collect all their definitions, and the definitions of various norms in one place. In general, we denote the norm on a function space $X$ by $\|\cdot\|_{X}$. However, more often used norms have a special notation, e.g., the inner product $(\cdot, \cdot)_{r}$ induces the norm $\|\cdot\|_{r}$ on $L_{r}^{2}(D)$, while the bilinear form $\Lambda(\cdot, \cdot)$ in (1.2) induces the norm

$$
\|\boldsymbol{v}\|_{r, \text { curl }}=\left(\|\boldsymbol{v}\|_{r}^{2}+\left\|\operatorname{curl}_{r z} \boldsymbol{v}\right\|_{r}^{2}\right)^{\frac{1}{2}}
$$

on $\boldsymbol{H}_{r}(\operatorname{curl}, D)$. Let $H_{r}^{k}(D)$, for any $k \geq 1$, be the space of all functions in $L_{r}^{2}(D)$ whose distributional derivatives up to order $k$ are in $L_{r}^{2}(D)$. The norm and seminorm on $H_{r}^{k}(D)$ are defined in the usual way. Let $\widetilde{H}_{r}^{1}(D)=L_{1 / r}^{2}(D) \cap H_{r}^{1}(D)$, where

$$
L_{1 / r}^{2}(D)=\left\{u \in L^{2}(D):\|u\|_{L_{1 / r}^{2}(D)}^{2}:=\int_{D} \frac{1}{r} u^{2} d r d z<\infty\right\} .
$$

Then

$$
\|v\|_{\widetilde{H}_{r}^{1}(D)}=\left(\|v\|_{H_{r}^{1}(D)}^{2}+\|v\|_{L_{1 / r}^{2}(D)}^{2}\right)^{\frac{1}{2}}
$$

defines a norm on $\widetilde{H}_{r}^{1}(D)$. Further, the seminorm and norm

$$
\begin{aligned}
|v|_{\widetilde{H}_{r}^{2}(D)} & =\left(\left|\frac{1}{r} \partial_{r}(r v)\right|_{H_{r}^{1}(D)}^{2}+\left|\partial_{z} v\right|_{H_{r}^{1}(D)}^{2}\right)^{\frac{1}{2}}, \\
\|v\|_{\widetilde{H}_{r}^{2}(D)} & =\left(|v|_{\widetilde{H}_{r}^{2}(D)}^{2}+\|v\|_{\widetilde{H}_{r}^{1}(D)}^{2}+\left\|\partial_{z} v\right\|_{L_{1 / r}^{2}(D)}^{2}\right)^{\frac{1}{2}}
\end{aligned}
$$


define the Hilbert space

$$
\widetilde{H}_{r}^{2}(D)=\left\{v \in \widetilde{H}_{r}^{1}(D):\|v\|_{\widetilde{H}_{r}^{2}(D)}<\infty\right\} .
$$

Functions in $\widetilde{H}_{r}^{1}(D)$ are well known to have zero trace on $\Gamma_{0}$ 3, 21. It is also known that functions in $H_{r}^{1}(D)$ have traces in $L_{r}^{2}\left(\Gamma_{1}\right)$, i.e., for $\phi$ in $H_{r}^{1}(D)$, the trace $\left.\phi\right|_{\Gamma_{1}}$ makes sense as a function in $L_{r}^{2}\left(\Gamma_{1}\right)$, but the trace on $\Gamma_{0}$ is not defined in general [23. Let $\mathcal{D}(D)$ denote the space of all infinitely differentiable functions that are compactly supported in $D$. Then we can define the tangential trace operator $\gamma_{t}: \mathcal{D}(D)^{2} \mapsto \widetilde{H}_{r}^{1}(D)^{\prime}$ by

$$
\left\langle\gamma_{t}(\boldsymbol{v}), \phi\right\rangle=\int_{\Gamma_{1}} r \boldsymbol{v} \cdot \boldsymbol{t} \phi d s \quad \text { for all } \phi \in \widetilde{H}_{r}^{1}(D),
$$

where $\langle\cdot, \cdot\rangle$ denotes duality pairing in $\widetilde{H}_{r}^{1}(D)$, and $\boldsymbol{t}$ is the unit tangent vector on $\partial D$, oriented counterclockwise. In [17. Proposition 2.2], it is shown that $\gamma_{t}$ extends to a continuous linear map from $\boldsymbol{H}_{r}(\operatorname{curl}, D)$ to $\widetilde{H}_{r}^{1}(D)^{\prime}$. Moreover, the integration by parts formula

$$
\left\langle\gamma_{t}(\boldsymbol{v}), \phi\right\rangle=\left(\boldsymbol{v}, \operatorname{curl}_{r z} \phi\right)_{r}-\left(\operatorname{curl}_{r z} \boldsymbol{v}, \phi\right)_{r}
$$

holds for all $\boldsymbol{v}$ in $\boldsymbol{H}_{r}(\operatorname{curl}, D)$ and $\phi$ in $\widetilde{H}_{r}^{1}(D)$. Here $\operatorname{curl}_{r z}$ is as defined in (1.4).

The boundary conditions for the 3D problem give rise to boundary conditions on $\Gamma_{1}$. We consider the perfect electric boundary condition on $\partial \Omega$, which asserts that the $3 \mathrm{D}$ electric field has zero tangential component on $\partial \Omega$. In the axisymmetric case, this boundary condition, loosely speaking, translates into an essential boundary condition of the form $\boldsymbol{E}_{r z} \cdot \boldsymbol{t}=0$ on $\Gamma_{1}$. More precisely, the weak solution will lie in the subspace of functions that are in the kernel of the tangential trace operator $\gamma_{t}$. Define

$$
\begin{aligned}
\boldsymbol{H}_{r, \diamond}(\operatorname{curl}, D) & =\left\{\boldsymbol{v} \in \boldsymbol{H}_{r}(\operatorname{curl}, D): \gamma_{t}(\boldsymbol{v})=0\right\} \\
H_{r, \diamond}^{1}(D) & =\left\{\phi \in H_{r}^{1}(D):\left.\phi\right|_{\Gamma_{1}}=0\right\}
\end{aligned}
$$

which are closed subspaces of $\boldsymbol{H}_{r}(\operatorname{curl}, D)$ and $H_{r}^{1}(D)$, respectively. In fact, the multigrid algorithm we shall give is for an equation posed in a subspace of $\boldsymbol{H}_{r, \diamond}(\operatorname{curl}, D)$. The case of $\boldsymbol{H}_{r}(\operatorname{curl}, D)$ without boundary conditions is similar and simpler.

We conclude this introduction with an outline of the remainder of the paper. There are a number of preliminary results we need before proceeding with the multigrid analysis. The first involves two commuting projectors with suitable approximation properties in weighted norms, which we present in the next section. In Section 3 we present and analyze an auxiliary mixed method which will prove to be a significant tool in our multigrid analysis of the positive definite problem related to the meridian problem. Although our primary interest in this paper is the meridian problem, the auxiliary mixed problem is in fact a weak variational problem of an azimuthal problem, and it is interesting in its own right. Section 4 describes the multigrid algorithm, and the succeeding section proves its uniform convergence. In Section [6] we present numerical results providing empirical support to the theoretical results. Finally, we summarize the results of the paper and discuss future work in Section 7. 


\section{Commuting Projectors}

The purpose of this section is to exhibit a projector $\Pi_{h}^{W}$ into the Nédélec finite element 25] subspace of $\boldsymbol{H}_{r, \diamond}(\operatorname{curl}, D)$ that has a commutativity property involving the $L_{r}^{2}(D)$-orthogonal projection $\Pi_{h}^{S}$ into a space of piecewise constant functions. We shall heavily use the commutativity and approximation properties of this projector in the next section.

First, let us define the finite element subspaces onto which the projections map. Let

$$
\begin{aligned}
\boldsymbol{N}_{1} & =\{(a-b z, c+b r): a, b, c \in \mathbb{R}\}, \\
P_{1} & =\left\{c_{0}+c_{1} r+c_{2} z: c_{i} \in \mathbb{R} \text { for } i=0,1,2\right\} .
\end{aligned}
$$

Assume that $D$ is meshed by a finite element triangulation $\mathscr{T}_{h}$ satisfying the usual geometrical conformity conditions [15. For simplicity, we assume that $\mathscr{T}_{h}$ is quasiuniform with a representative mesh size $h$. The finite element spaces we shall use are

$$
\begin{aligned}
V_{h} & =\left\{u \in H_{r}^{1}(D):\left.u\right|_{K} \in P_{1} \text { for all } K \in \mathscr{T}_{h}\right\}, \\
\boldsymbol{W}_{h} & =\left\{\boldsymbol{v} \in \boldsymbol{H}_{r}(\operatorname{curl}, D):\left.\boldsymbol{v}\right|_{K} \in \boldsymbol{N}_{1} \text { for all } K \in \mathscr{T}_{h}\right\}, \\
V_{h, \diamond} & =\left\{v \in V_{h}:\left.v\right|_{\Gamma_{1}}=0\right\}, \\
\boldsymbol{W}_{h, \diamond} & =\left\{\boldsymbol{v} \in \boldsymbol{W}_{h}: \gamma_{t}(\boldsymbol{v})=0\right\}, \\
S_{h} & =\left\{u \in L_{r}^{2}(D):\left.u\right|_{K} \text { is constant for all } K \in \mathscr{T}_{h}\right\} .
\end{aligned}
$$

Projectors into these finite element spaces with commutativity properties have been constructed previously. Indeed, in [17], we find projectors $\widehat{\Pi}_{h}^{V}, \widehat{\Pi}_{h}^{W}$, and $\widehat{\Pi}_{h}^{S}$ onto $V_{h, \diamond}, \boldsymbol{W}_{h, \diamond}$, and $S_{h}$, respectively. In particular, it is proved in [17] that they satisfy

$$
\begin{gathered}
\operatorname{curl}_{r z} \widehat{\Pi}_{h}^{W} \boldsymbol{v}=\widehat{\Pi}_{h}^{S} \operatorname{curl}_{r z} \boldsymbol{v}, \\
\left\|\widehat{\Pi}_{h}^{W} \boldsymbol{v}-\boldsymbol{v}\right\|_{r} \leq C h|\boldsymbol{v}|_{H_{r}^{1}(D)}
\end{gathered}
$$

for all $\boldsymbol{v}$ in $H_{r}^{1}(D)$ (see [17, Lemma 5.1] for (2.1) and [17, Lemma 5.3] for (2.2)). The projection $\widehat{\Pi}_{h}^{S} \phi$ equals the $L_{r}^{2}(K)$-orthogonal projection of $\phi$ for all $K$ intersecting $\Gamma_{0}$, while for the remaining elements $K^{\prime}$, it equals the (unweighted) $L^{2}\left(K^{\prime}\right)$ orthogonal projection of $\phi$.

Unfortunately, these projectors are inadequate for our purposes in this paper. Let $\Pi_{h}^{S}$ denote the $L_{r}^{2}(D)$-orthogonal projection into $S_{h}$. For our analysis later (in particular, in the proof of Theorem 3.3), we need a projector $\Pi_{h}^{W}$ that satisfies the commutativity property in (2.1) with $\Pi_{h}^{S}$. The projector $\widehat{\Pi}_{h}^{S}$ of [17] is not equal to $\Pi_{h}^{S}$. Therefore, the remainder of this section is devoted to the construction of the projector $\Pi_{h}^{W}$ with the properties we need, as listed in the following theorem.

Theorem 2.1. Let $\Pi_{h}^{S}: L_{r}^{2}(D) \rightarrow S_{h}$ be the $L_{r}^{2}(D)$-orthogonal projection. There is a projector $\Pi_{h}^{W}: \boldsymbol{H}_{r, \diamond}(\operatorname{curl}, D) \rightarrow \boldsymbol{W}_{h, \diamond}$ such that

(1) $\Pi_{h}^{W}$ is well defined and continuous on $\boldsymbol{H}_{r, \diamond}(\operatorname{curl}, D)$,

(2) the commutativity property

$$
\Pi_{h}^{S} \operatorname{curl}_{r z} \boldsymbol{u}=\operatorname{curl}_{r z} \Pi_{h}^{W} \boldsymbol{u}
$$

holds for all $\boldsymbol{u}$ in $\boldsymbol{H}_{r, \diamond}(\operatorname{curl}, D)$,

(3) the approximation property

$$
\left\|\boldsymbol{u}-\Pi_{h}^{W} \boldsymbol{u}\right\|_{r, \mathrm{curl}} \leq C \inf _{\boldsymbol{u}_{h} \in \boldsymbol{W}_{h, \diamond}}\left\|\boldsymbol{u}-\boldsymbol{u}_{h}\right\|_{r, \text { curl }}
$$


holds for all $\boldsymbol{u}$ in $\boldsymbol{H}_{r, \diamond}(\operatorname{curl}, D)$.

Above, and in the rest of the paper, we use $C$ to denote a generic constant independent of the functions involved in the norm estimates, which may take different values at different occurrences. We remark that the projector $\Pi_{h}^{W}$ is continuous on $\boldsymbol{H}_{r, \diamond}(\operatorname{curl}, D)$, whereas typical projectors into the Nédélec space, such as Nédélec's original projector [25], as well as the projector $\widehat{\Pi}_{h}^{W}$, require more regularity due to edge-based degrees of freedom. This is achieved by a global definition of $\Pi_{h}^{W}$ without local degrees of freedom, given in the proof of this theorem below.

For the proof and subsequent analysis, we will need an "exact sequence property" and the so-called "discrete Helmholtz decomposition," but adapted to our weighted inner product setting. Because we have assumed that $\Gamma_{1}$ is connected and $D$ is simply connected, it follows that the sequence

$$
0 \longrightarrow V_{h, \diamond} \stackrel{\operatorname{grad}_{r z}}{\longrightarrow} \boldsymbol{W}_{h, \diamond} \stackrel{\operatorname{curl}_{r z}}{\longrightarrow} S_{h} \longrightarrow 0
$$

is exact, as proved in Appendix A. This means that the map $\operatorname{curl}_{r z}: \boldsymbol{W}_{h, \diamond} \mapsto S_{h}$ is surjective and its null space coincides with the $\operatorname{grad}_{r z}\left(V_{h, \diamond}\right)$. Such results are standard in the case of no boundary conditions or when boundary conditions hold on the entire boundary. In our application, a boundary condition is prescribed only on part of $\partial D$, namely $\Gamma_{1}$. Since we have not been able to locate a reference for the proof of exactness in this case, we include a short proof in Appendix $\mathrm{A}$.

Next, let us adapt the well-known discrete Helmholtz decomposition to our weighted norms. Given a $\boldsymbol{v}_{h}$ in $\boldsymbol{W}_{h, \diamond}$, there is a unique $\phi_{h}$ in $V_{h, \diamond}$ satisfying

$$
\left(\operatorname{grad}_{r z} \phi_{h}, \operatorname{grad}_{r z} \psi_{h}\right)_{r}=\left(\boldsymbol{v}_{h}, \operatorname{grad}_{r z} \psi_{h}\right)_{r} \quad \text { for all } \psi_{h} \text { in } V_{h, \diamond} \text {. }
$$

The unique existence of $\phi_{h}$ is guaranteed by the Lax-Milgram lemma, which may be invoked for this variational problem thanks to [21, Proposition 2.1]. It is trivial to verify the stability estimate

$$
\left\|\operatorname{grad}_{r z} \phi_{h}\right\|_{r} \leq\left\|\boldsymbol{v}_{h}\right\|_{r} .
$$

Let $\boldsymbol{r}_{h}=\boldsymbol{v}_{h}-\operatorname{grad}_{r z} \phi_{h}$. Then the weighted discrete Helmholtz decomposition is

$$
\boldsymbol{v}_{h}=\operatorname{grad}_{r z} \phi_{h}+\boldsymbol{r}_{h} .
$$

Note that the components of the decomposition are orthogonal with respect to the weighted inner products of both $L_{r}^{2}(D)^{2}$ and $\boldsymbol{H}_{r}(\operatorname{curl}, D)$.

To characterize $\boldsymbol{r}_{h}$ further, let $\operatorname{curl}_{r z}^{\prime}: S_{h} \mapsto \boldsymbol{W}_{h, \diamond}$ be defined by

$$
\left(\operatorname{curl}_{r z}^{\prime} s_{h}, \boldsymbol{w}_{h}\right)_{r}=\left(s_{h}, \operatorname{curl}_{r z} \boldsymbol{w}_{h}\right)_{r}, \quad \text { for all } s_{h} \text { in } S_{h} \text { and } \boldsymbol{w}_{h} \text { in } \boldsymbol{W}_{h, \diamond},
$$

i.e., $\operatorname{curl}_{r z}^{\prime}$ is the adjoint of $\operatorname{curl}_{r z}: \boldsymbol{W}_{h, \diamond} \rightarrow S_{h}$, with respect to the weighted inner product $(\cdot, \cdot)_{r}$. By the exactness of (2.3),

$$
\operatorname{grad}_{r z}\left(V_{h, \diamond}\right)=\operatorname{ker}\left(\operatorname{curl}_{r z}\right) \text {, }
$$

where $\operatorname{ker}\left(\operatorname{curl}_{r z}\right)$ denotes the null space of $\operatorname{curl}_{r z}$ in $\boldsymbol{W}_{h, \diamond}$. Hence the orthogonality of $\boldsymbol{r}_{h}$ with $\operatorname{grad}_{r z}\left(V_{h, \diamond}\right)$ implies that $\boldsymbol{r}_{h}$ is in the range of the adjoint curl ${ }_{r z}^{\prime}$, i.e., there is an element $a_{h}$ in $S_{h}$ such that

$$
\boldsymbol{r}_{h}=\operatorname{curl}_{r z}^{\prime} a_{h},
$$

and moreover, $a_{h}$ is unique due to the injectivity of $\operatorname{curl}_{r z}^{\prime}$ (which follows from the surjectivity of $\operatorname{curl}_{r z}$ in the exact sequence (2.3) ). In other words, an alternate way 
of stating the decomposition is that for all $\boldsymbol{v}_{h}$ in $\boldsymbol{W}_{h, \diamond}$, there is a unique $a_{h}$ in $S_{h}$ and a unique $\phi_{h}$ in $V_{h, \diamond}$ such that

$$
\boldsymbol{v}_{h}=\operatorname{grad}_{r z} \phi_{h}+\operatorname{curl}_{r z}^{\prime} a_{h} .
$$

We shall now use this decomposition to prove Theorem 2.1

Proof of Theorem 2.1. Define $\Pi_{h}^{W}: \boldsymbol{H}_{r, \diamond}(\operatorname{curl}, D) \rightarrow \boldsymbol{W}_{h, \diamond}$ by

$$
\begin{aligned}
\left(\Pi_{h}^{W} \boldsymbol{v}, \operatorname{grad}_{r z} \eta_{h}\right)_{r} & =\left(\boldsymbol{v}, \operatorname{grad}_{r z} \eta_{h}\right)_{r} & & \text { for all } \eta_{h} \text { in } V_{h, \diamond}, \\
\left(\operatorname{curl}_{r z} \Pi_{h}^{W} \boldsymbol{v}, s_{h}\right)_{r} & =\left(\operatorname{curl}_{r z} \boldsymbol{v}, s_{h}\right)_{r} & & \text { for all } s_{h} \text { in } S_{h} .
\end{aligned}
$$

We will now verify that the asserted statements hold for this $\Pi_{h}^{W}$.

(11) First of all, observe that (2.6) is a square system of equations. Indeed, due to the exactness of (2.3), the number of equations in (2.6) equals

$$
\begin{aligned}
\operatorname{dim}\left(V_{h, \diamond}\right)+\operatorname{dim}\left(S_{h}\right) & =\operatorname{dim}\left(\operatorname{grad}_{r z}\left(V_{h, \diamond}\right)\right)+\operatorname{dim}\left(\operatorname{curl}_{r z}\left(\boldsymbol{W}_{h, \diamond}\right)\right) \\
& =\operatorname{dim}\left(\operatorname{ker}\left(\operatorname{curl}_{r z}\right)\right)+\operatorname{dim}\left(\operatorname{curl}_{r z}\left(\boldsymbol{W}_{h, \diamond}\right)\right) \\
& =\operatorname{dim}\left(\boldsymbol{W}_{h, \diamond}\right)
\end{aligned}
$$

by the rank-nullity theorem. Thus, we only need to show that the kernel of the linear system (2.6) is trivial. If $\boldsymbol{v}=0$, then the right hand side of (2.6) is zero, so

$$
\left(\Pi_{h}^{W} \boldsymbol{v}, \operatorname{grad}_{r z} \eta_{h}+\operatorname{curl}_{r z}^{\prime} s_{h}\right)_{r}=0 \quad \text { for all } \eta_{h} \text { in } V_{h, \diamond} \text { and } s_{h} \text { in } S_{h} .
$$

This implies that $\Pi_{h}^{W} \boldsymbol{v}=0$, by the weighted discrete Helmholtz decomposition of $\boldsymbol{W}_{h, \diamond}$. Therefore, $\Pi_{h}^{W}$ is well defined.

Before we proceed to prove the continuity of $\Pi_{h}^{W}$ on $\boldsymbol{H}_{r, \diamond}(\operatorname{curl}, D)$, let us note that

$$
\left\|s_{h}\right\|_{r} \leq C\left\|\operatorname{curl}_{r z}^{\prime} s_{h}\right\|_{r} \quad \text { for all } s_{h} \in S_{h} .
$$

This follows from [17. Theorem 6.1(2)], which asserts that

$$
\left\|\boldsymbol{v}_{h}\right\|_{r} \leq C\left\|\operatorname{curl}_{r z} \boldsymbol{v}_{h}\right\|_{r} \quad \text { for all } \boldsymbol{v}_{h} \in \boldsymbol{R}_{h}^{\perp},
$$

where $\boldsymbol{R}_{h}^{\perp}$ denotes the orthogonal complement of $\operatorname{grad}_{r z}\left(V_{h, \diamond}\right)$ in $\boldsymbol{W}_{h, \diamond}$ in the weighted $L_{r}^{2}(D)$-norm. Indeed, (2.8) implies that

$$
\left\|\operatorname{curl}_{r z}^{\prime} a_{h}\right\|_{r}=\sup _{\boldsymbol{v}_{h} \in \boldsymbol{R}_{h}^{\perp}} \frac{\left(\operatorname{curl}_{r z}^{\prime} a_{h}, \boldsymbol{v}_{h}\right)_{r}}{\left\|\boldsymbol{v}_{h}\right\|_{r}} \geq \sup _{\boldsymbol{v}_{h} \in \boldsymbol{R}_{h}^{\perp}} \frac{\left(a_{h}, \operatorname{curl}_{r z} \boldsymbol{v}_{h}\right)_{r}}{C\left\|\operatorname{curl}_{r z} \boldsymbol{v}_{h}\right\|_{r}}=\frac{1}{C}\left\|a_{h}\right\|_{r},
$$

which proves (2.7).

Now, to prove the continuity of $\Pi_{h}^{W}$, let us first use the weighted discrete Helmholtz decomposition (2.5) and write $\Pi_{h}^{W} \boldsymbol{v}=\operatorname{grad}_{r z} \phi_{h}+\operatorname{curl}_{r z}^{\prime} a_{h}$, with $\phi_{h}$ in $V_{h, \diamond}$ and $a_{h}$ in $S_{h}$. Setting $\eta_{h}=\phi_{h}$ in (2.6a) and $s_{h}=a_{h}$ in (2.6b) and applying the Cauchy-Schwarz inequality,

$$
\begin{aligned}
\left\|\operatorname{grad}_{r z} \phi_{h}\right\|_{r} & \leq\|\boldsymbol{v}\|_{r}, \quad \text { and } \\
\left\|\operatorname{curl}_{r z}^{\prime} a_{h}\right\|_{r}^{2} & =\left(\operatorname{curl}_{r z} \boldsymbol{v}, a_{h}\right)_{r} \leq\left\|\operatorname{curl}_{r z} \boldsymbol{v}\right\|_{r}\left\|a_{h}\right\|_{r} \\
& \leq\left\|\operatorname{curl}_{r z} \boldsymbol{v}\right\|_{r} C\left\|\operatorname{curl}_{r z}^{\prime} a_{h}\right\|_{r}
\end{aligned}
$$

by (2.7). Hence, the stated continuity of $\Pi_{h}^{W}$ follows by the stability of the weighted discrete Helmholtz decomposition.

(2) Commutativity is clear from (2.6b) and the definition of $\Pi_{h}^{S}$. 
(3) To prove the error estimate, consider an arbitrary $\boldsymbol{u}$ in $\boldsymbol{H}_{r, \diamond}(\operatorname{curl}, D)$ and $\boldsymbol{u}_{h}$ in $\boldsymbol{W}_{h, \diamond}$. Use the weighted discrete Helmholtz decomposition to split

$$
\Pi_{h}^{W} \boldsymbol{u}-\boldsymbol{u}_{h}=\operatorname{grad}_{r z} \psi_{h}+\operatorname{curl}_{r z}^{\prime} b_{h},
$$

with $\psi_{h}$ in $V_{h, \diamond}$ and $b_{h}$ in $S_{h}$. Then, by (2.6),

$$
\begin{aligned}
\left(\Pi_{h}^{W} \boldsymbol{u}-\boldsymbol{u}, \Pi_{h}^{W} \boldsymbol{u}-\boldsymbol{u}_{h}\right)_{r} & =\left(\Pi_{h}^{W} \boldsymbol{u}-\boldsymbol{u}, \operatorname{grad}_{r z} \psi_{h}+\operatorname{curl}_{r z}^{\prime} b_{h}\right)_{r} \\
& =\left(\Pi_{h}^{W} \boldsymbol{u}-\boldsymbol{u}, \operatorname{curl}_{r z}^{\prime} b_{h}\right)_{r} \\
& =\left(\operatorname{curl}_{r z} \Pi_{h}^{W} \boldsymbol{u}, b_{h}\right)_{r}-\left(\boldsymbol{u}_{h}, \operatorname{curl}_{r z}^{\prime} b_{h}\right)_{r}-\left(\boldsymbol{u}-\boldsymbol{u}_{h}, \operatorname{curl}_{r z}^{\prime} b_{h}\right)_{r} \\
& =\left(\operatorname{curl}_{r z} \boldsymbol{u}, b_{h}\right)_{r}-\left(\operatorname{curl}_{r z} \boldsymbol{u}_{h}, b_{h}\right)_{r}-\left(\boldsymbol{u}-\boldsymbol{u}_{h}, \operatorname{curl}_{r z}^{\prime} b_{h}\right)_{r}
\end{aligned}
$$

and hence

$$
\left\|\Pi_{h}^{W} \boldsymbol{u}-\boldsymbol{u}_{h}\right\|_{r}^{2}=\left(\boldsymbol{u}-\boldsymbol{u}_{h}, \Pi_{h}^{W} \boldsymbol{u}-\boldsymbol{u}_{h}\right)_{r}+\left(\operatorname{curl}_{r z}\left(\boldsymbol{u}-\boldsymbol{u}_{h}\right), b_{h}\right)_{r}-\left(\boldsymbol{u}-\boldsymbol{u}_{h}, \operatorname{curl}_{r z}^{\prime} b_{h}\right)_{r} .
$$

Now, using the Cauchy-Schwarz inequality,

$$
\begin{aligned}
\left\|\Pi_{h}^{W} \boldsymbol{u}-\boldsymbol{u}_{h}\right\|_{r}^{2} & \leq\left\|\boldsymbol{u}-\boldsymbol{u}_{h}\right\|_{r, \text { curl }}\left(\left\|\Pi_{h}^{W} \boldsymbol{u}-\boldsymbol{u}_{h}\right\|_{r}+\left\|b_{h}\right\|_{r}+\left\|\operatorname{curl}_{r z}^{\prime} b_{h}\right\|_{r}\right) \\
& \leq C\left\|\boldsymbol{u}-\boldsymbol{u}_{h}\right\|_{r, \text { curl }}\left\|\Pi_{h}^{W} \boldsymbol{u}-\boldsymbol{u}_{h}\right\|_{r}
\end{aligned}
$$

where we have applied (2.7) to $b_{h}$, and used the stability of the discrete Helmholtz decomposition. The triangle inequality now yields the estimate

$$
\left\|\boldsymbol{u}-\Pi_{h}^{W} \boldsymbol{u}\right\|_{r} \leq C\left\|\boldsymbol{u}-\boldsymbol{u}_{h}\right\|_{r, \mathrm{curl}} .
$$

Finally, since

$$
\left\|\operatorname{curl}_{r z}\left(\boldsymbol{u}-\Pi_{h}^{W} \boldsymbol{u}\right)\right\|_{r}=\left\|\operatorname{curl}_{r z} \boldsymbol{u}-\Pi_{h}^{S} \operatorname{curl}_{r z} \boldsymbol{u}\right\|_{r} \leq\left\|\operatorname{curl}_{r z} \boldsymbol{u}-\operatorname{curl}_{r z} \boldsymbol{u}_{h}\right\|_{r}
$$

we have

$$
\left\|\boldsymbol{u}-\Pi_{h}^{W} \boldsymbol{u}\right\|_{r, \operatorname{curl}} \leq C\left\|\boldsymbol{u}-\boldsymbol{u}_{h}\right\|_{r, \mathrm{curl}}
$$

Since $\boldsymbol{u}_{h}$ in $\boldsymbol{W}_{h, \diamond}$ is arbitrary, this proves the stated approximation property.

The following corollary gives more specific estimates in terms of the meshsize $h$, under certain conditions.

Corollary 2.1. Let $\boldsymbol{u}$ be in $H_{r}^{1}(D)^{2}$. The approximation property

$$
\left\|\boldsymbol{u}-\Pi_{h}^{W} \boldsymbol{u}\right\|_{r, \operatorname{curl}} \leq C h\left(|\boldsymbol{u}|_{H_{r}^{1}(D)^{2}}+\left|\operatorname{curl}_{r z} \boldsymbol{u}\right|_{H_{r}^{1}(D)}\right)
$$

holds provided that $\operatorname{curl}_{r z} \boldsymbol{u}$ is in $H_{r}^{1}(D)$, and

$$
\left\|\boldsymbol{u}-\Pi_{h}^{W} \boldsymbol{u}\right\|_{r} \leq C h|\boldsymbol{u}|_{H_{r}^{1}(D)^{2}}
$$

holds provided that $\operatorname{curl}_{r z} \boldsymbol{u}$ is in $S_{h}$.

Proof. In the case that $\operatorname{curl}_{r z} \boldsymbol{u}$ is in $H_{r}^{1}(D)$, the first estimate follows directly from Theorem 2.1(3) and (2.2), by taking $\boldsymbol{u}_{h}=\widehat{\Pi}_{h}^{W} \boldsymbol{u}$.

Now suppose $\operatorname{curl}_{r z} \boldsymbol{u}$ is in $S_{h}$. Then the following estimate of Theorem 2.1(3),

$$
\left\|\boldsymbol{u}-\Pi_{h}^{W} \boldsymbol{u}\right\|_{r, \operatorname{curl}} \leq C\left\|\boldsymbol{u}-\widehat{\Pi}_{h}^{W} \boldsymbol{u}\right\|_{r, \operatorname{curl}}
$$

reduces to simply

$$
\left\|\boldsymbol{u}-\Pi_{h}^{W} \boldsymbol{u}\right\|_{r} \leq C\left\|\boldsymbol{u}-\widehat{\Pi}_{h}^{W} \boldsymbol{u}\right\|_{r}
$$

because of the commutativity properties. Indeed, $\operatorname{curl}_{r z} \widehat{\Pi}_{h}^{W} \boldsymbol{u}=\widehat{\Pi}_{h}^{S} \operatorname{curl}_{r z} \boldsymbol{u}=$ $\operatorname{curl}_{r z} \boldsymbol{u}$ and similarly $\operatorname{curl}_{r z} \Pi_{h}^{W} \boldsymbol{u}=\operatorname{curl}_{r z} \boldsymbol{u}$. Using (2.2), the estimate (2.9) then follows. 


\section{A DUAL MIXED PROBLEM}

In our multigrid analysis presented later, we will use an intermediate mixed variational problem to obtain several estimates. This mixed problem, which is interesting in its own right, is the subject of the present section. We will prove that the problem is well posed and provide error estimates for the discrete solution.

The problem can be stated as follows: Find $\boldsymbol{z}$ in $\boldsymbol{H}_{r, \diamond}(\operatorname{curl}, D)$ and $p$ in $L_{r}^{2}(D)$ satisfying

$$
\begin{aligned}
& (\boldsymbol{z}, \boldsymbol{w})_{r}-\left(p, \operatorname{curl}_{r z} \boldsymbol{w}\right)_{r}=0, \quad \text { for all } \boldsymbol{w} \text { in } \boldsymbol{H}_{r, \diamond}(\operatorname{curl}, D), \\
& \left(s, \operatorname{curl}_{r z} \boldsymbol{z}\right)_{r}=(s, f)_{r}, \quad \text { for all } s \text { in } L_{r}^{2}(D) .
\end{aligned}
$$

Observe that this is a variational formulation of the boundary value problem:

$$
\begin{aligned}
\boldsymbol{z} & =\operatorname{curl}_{r z} p & & \text { on } D, \\
\operatorname{curl}_{r z} \boldsymbol{z} & =f & & \text { on } D, \\
\boldsymbol{z} \cdot \boldsymbol{t} & =0 & & \text { on } \Gamma_{1},
\end{aligned}
$$

which can also be written as the second-order boundary value problem

$$
\operatorname{curl}_{r z} \operatorname{curl}_{r z} p=f \quad \text { on } D \quad \text { and } \quad \operatorname{curl}_{r z} p \cdot \mathbf{t}=0 \quad \text { on } \Gamma_{1} .
$$

The differential operator appearing here is the same as the second-order operator appearing in the equation defining the azimuthal component $\left(E_{\theta}\right)$ of the axisymmetric Maxwell equations (for a homogeneous medium). Interestingly, this "azimuthal" operator plays an important role in the multigrid analysis of the "meridian" operator. Problem (3.1) is independently interesting because of the above-mentioned connection to the azimuthal Maxwell system. Indeed, a primal variational formulation of (3.2), but with different boundary conditions, is analyzed in [21. In this section, we will analyze the dual variational formulation (3.1).

We begin with the following lemma, which will help in proving that the mixed problem (3.1) is well posed (cf. Lemma A.1 in Appendix A).

Lemma 3.1. The map $\operatorname{curl}_{r z}: \boldsymbol{H}_{r, \diamond}(\operatorname{curl}, D) \mapsto L_{r}^{2}(D)$ is surjective.

Proof. Let $s$ be in $L_{r}^{2}(D)$. It is shown in [5, 21] that there exists a unique $u$ in $V^{\theta}$ satisfying

$$
\left(\operatorname{curl}_{r z} u, \operatorname{curl}_{r z} v\right)_{r}=(s, v)_{r} \quad \text { for all } v \in V^{\theta},
$$

where $V^{\theta}:=\left\{v \in \widetilde{H}_{r}^{1}(D): v=0\right.$ on $\left.\partial D\right\}$. This implies that $s=\operatorname{curl}_{r z} \operatorname{curl}_{r z} u$ in $L_{r}^{2}(D)$, by (1.6) and the density of $\mathcal{D}(D)$ in $L_{r}^{2}(D)$. Hence, setting $\boldsymbol{w}=\operatorname{curl}_{r z} u$, we find that

$$
s=\operatorname{curl}_{r z} \boldsymbol{w} .
$$

Note that $\boldsymbol{w}$ is in $L_{r}^{2}(D)^{2}$, since $\left\|\operatorname{curl}_{r z} u\right\|_{r} \leq C\|u\|_{\widetilde{H}_{r}^{1}(D)}$ by [21, Proposition 3.1]. In fact, these two norms are equivalent as $u$ is in $V^{\theta}$. Moreover, by (3.4), $\operatorname{curl}_{r z} \boldsymbol{w}$ is in $L_{r}^{2}(D)$, so $\boldsymbol{w}$ is in $\boldsymbol{H}_{r}(\operatorname{curl}, D)$. However, we want to express $s$ as the curl of a function in $\boldsymbol{H}_{r, \diamond}(\operatorname{curl}, D)$. To this end, we first define $\boldsymbol{W}^{0}$ by

$$
\boldsymbol{W}^{0}:=\left\{\boldsymbol{w} \in \boldsymbol{H}_{r, \diamond}(\operatorname{curl}, D):\left(\boldsymbol{w}, \operatorname{grad}_{r z} q\right)_{r}=0 \text { for all } q \in H_{r, \diamond}^{1}(D)\right\}
$$

and let $P^{0}$ denote the orthogonal projection from $\boldsymbol{H}_{r}(\operatorname{curl}, D)$ to $\boldsymbol{W}^{0}$ in the $(\cdot, \cdot)_{r^{-}}$ inner product. Clearly, if we show that

$$
s=\operatorname{curl}_{r z}\left(P^{0} \boldsymbol{w}\right),
$$


the proof of the lemma will be complete.

Considering any $\psi$ in $\mathcal{D}(D)$, it is easy to check that

$$
\operatorname{curl}_{r z} \psi \text { is in } \boldsymbol{W}^{0} \text {. }
$$

Furthermore, since $\psi$ is in $\mathcal{D}(D)$,

$$
\begin{aligned}
\left(\operatorname{curl}_{r z}\left(P^{0} \boldsymbol{w}\right), \psi\right)_{r} & =\left(P^{0} \boldsymbol{w}, \operatorname{curl}_{r z} \psi\right)_{r} & & \text { by (1.6), } \\
& =\left(\boldsymbol{w}, \operatorname{curl}_{r z} \psi\right)_{r} & & \text { by (3.6), } \\
& =(s, \psi)_{r} & & \text { by (1.6) and (3.4). }
\end{aligned}
$$

Since $\mathcal{D}(D)$ is dense in $L_{r}^{2}(D)$ (see e.g. 23]), we have proved (3.5).

Theorem 3.1. There exists a unique $\boldsymbol{z}$ in $\boldsymbol{H}_{r, \diamond}(\operatorname{curl}, D)$ and a unique $p$ in $L_{r}^{2}(D)$ satisfying (3.1). Moreover, there is a constant $C_{\text {stability }}>0$ independent of $f$ such that

$$
\|\boldsymbol{z}\|_{r, \text { curl }}+\|p\|_{r} \leq C_{\text {stability }}\|f\|_{r} .
$$

Proof. By the Babuška-Brezzi theory of mixed methods [13, the theorem will follow once we verify the inf-sup condition

$$
C_{1}\|s\|_{r} \leq \sup _{\boldsymbol{v} \in \boldsymbol{H}_{r, \diamond}(\operatorname{curl}, D)} \frac{\left(\operatorname{curl}_{r z} \boldsymbol{v}, s\right)_{r}}{\|\boldsymbol{v}\|_{r, \text { curl }}} \quad \text { for all } s \text { in } L_{r}^{2}(D),
$$

and coercivity on the kernel,

$$
\|\boldsymbol{v}\|_{r, \text { curl }} \leq C_{2}\|\boldsymbol{v}\|_{r} \quad \text { for all } \boldsymbol{v} \text { in } \boldsymbol{G},
$$

where $\boldsymbol{G}$ is the kernel defined by

$$
\boldsymbol{G}=\left\{\boldsymbol{w} \in \boldsymbol{H}_{r, \diamond}(\operatorname{curl}, D):\left(\operatorname{curl}_{r z} \boldsymbol{w}, s\right)_{r}=0 \text { for all } s \text { in } L_{r}^{2}(D)\right\} .
$$

Above, $C_{1}$ and $C_{2}$ are two constants independent of the functions involved.

The inf-sup condition (3.7) is equivalent to asserting that the adjoint of the operator

$$
\operatorname{curl}_{r z}: \boldsymbol{H}_{r, \diamond}(\operatorname{curl}, D) \mapsto L_{r}^{2}(D)
$$

is bounded from below, which is equivalent to the surjectivity of the above curl map (by standard arguments using the Closed Range Theorem; see e.g., [13, § II.1]). This surjectivity is precisely the assertion of Lemma 3.1. Hence, it only remains to verify (3.8), which is obvious, since $\boldsymbol{v}$ is in $\boldsymbol{G}$ if and only if $\operatorname{curl}_{r z} \boldsymbol{v}=0$, so that $\|\boldsymbol{v}\|_{r, \text { curl }}=\|\boldsymbol{v}\|_{r}$ for all $\boldsymbol{v}$ in $\boldsymbol{G}$.

Next, we prove a regularity result under the assumption that the revolution of $D$ about the axis of symmetry, namely $\Omega$, is convex. Denote by $\breve{L}^{2}(\Omega)$ and $\breve{H}^{k}(\Omega)$ the subspaces of axisymmetric functions of $L^{2}(\Omega)$ and $H^{k}(\Omega)$, respectively, for $k \geq 1$. The restriction map $g(r, \theta, z) \mapsto g_{D}(r, z)$, given by

$$
g_{D}(r, z)=g(r, 0, z), \text { for all }(r, z) \text { in } D,
$$

is an isometry (up to a factor of $\sqrt{2 \pi}$ ) from $\breve{L}^{2}(\Omega)$ onto $L_{r}^{2}(D)$. The reverse operation will be denoted by superscripting functions with $\Omega$, i.e., given $\eta(r, z)$ on $D$, the function $\eta^{\Omega}$ is defined by $\eta^{\Omega}(r, \theta, z)=\eta(r, z)$. Thus $\left(g_{D}\right)^{\Omega}=g$, for $g$ in $\breve{L}^{2}(\Omega)$.

With the use of such notation, we will now prove the following estimates, which will be useful in our multigrid analysis. 
Theorem 3.2. If the revolution of $D$ is convex, then there is a constant $C_{\text {regularity }}>$ 0 such that the solution $(\boldsymbol{z}, p)$ of (3.1) satisfies

$$
\begin{aligned}
\|\boldsymbol{z}\|_{H_{r}^{1}(D)^{2}} & \leq C_{\text {regularity }}\|f\|_{r}, \\
\|p\|_{\widetilde{H}_{r}^{2}(D)} & \leq C_{\text {regularity }}\|f\|_{r},
\end{aligned}
$$

for any data $f$ in $L_{r}^{2}(D)$.

Proof. Let $(\boldsymbol{z}, p)$ solve (3.1). Define $\boldsymbol{p}_{\theta}=p^{\Omega} \boldsymbol{e}_{\theta}$. Then recalling the expression

$$
\operatorname{div} \boldsymbol{q}=\frac{1}{r} \partial_{r}\left(r q_{r}\right)+\frac{1}{r} \partial_{\theta} q_{\theta}+\partial_{z} q_{z}
$$

for divergence in cylindrical coordinates, we find that

$$
\begin{array}{ll}
\operatorname{div} \boldsymbol{p}_{\theta}=0 & \text { on } \Omega, \\
\boldsymbol{p}_{\theta} \cdot \boldsymbol{n}=0 & \text { on } \partial \Omega .
\end{array}
$$

The last equality follows because the unit outward normal $\boldsymbol{n}$ on $\partial \Omega$ is orthogonal to $\boldsymbol{e}_{\theta}$. Furthermore, from the first equation of (3.1), we know that the equality $\boldsymbol{z}-\operatorname{curl}_{r z} p=0$ holds in the distributional sense. Since $\boldsymbol{z}$ is in $L_{r}^{2}(D)^{2}$, the equality

$$
z=\operatorname{curl}_{r z} p
$$

in fact holds in $L_{r}^{2}(D)^{2}$. By writing out the three-dimensional curl in cylindrical coordinates, we observe that

$$
\operatorname{curl} p_{\theta}=\left(\operatorname{curl}_{r z} p\right)^{\Omega}=\boldsymbol{z}^{\Omega},
$$

where for axisymmetric vector fields $\boldsymbol{v}=v_{r} \boldsymbol{e}_{r}+v_{z} \boldsymbol{e}_{z}$, the revolution is defined by $\boldsymbol{v}^{\Omega}=v_{r}^{\Omega} \boldsymbol{e}_{r}+v_{z}^{\Omega} \boldsymbol{e}_{z}$. Thus curl $\boldsymbol{p}_{\theta}$ is in $L^{2}(\Omega)^{3}$. Combining these observations, we find that $\boldsymbol{p}_{\theta}$ is in $\boldsymbol{H}(\operatorname{curl}, \Omega) \cap \boldsymbol{H}_{0}(\operatorname{div}, \Omega)$, a space which is well known to be continuously imbedded in $\boldsymbol{H}^{1}(\Omega)$ whenever $\Omega$ is convex [20]. Thus

$$
\begin{aligned}
\left\|\boldsymbol{p}_{\theta}\right\|_{\boldsymbol{H}^{1}(\Omega)} & \leq C\left(\left\|\boldsymbol{p}_{\theta}\right\|_{\boldsymbol{H}(\operatorname{curl}, \Omega)}+\left\|\boldsymbol{p}_{\theta}\right\|_{\boldsymbol{H}(\operatorname{div}, \Omega)}\right) \\
& \leq C\left(\|p\|_{L_{r}^{2}(D)}+\left\|\operatorname{curl}_{r z} p\right\|_{L_{r}^{2}(D)^{2}}\right) \\
& \leq C\left(\|p\|_{L_{r}^{2}(D)}+\|\boldsymbol{z}\|_{L_{r}^{2}(D)^{2}}\right) \\
& \leq C\|f\|_{r},
\end{aligned}
$$

where in the last step we have used Theorem 3.1

The second equality of the variational problem (3.1) shows that $\operatorname{curl}_{r z} z=f$ holds in $L_{r}^{2}(D)$. Translating this for $\boldsymbol{z}^{\Omega}$, we have

$$
\begin{aligned}
\operatorname{curl} \boldsymbol{z}^{\Omega} & =f^{\Omega} \boldsymbol{e}_{\theta} & & \text { on } \Omega, \\
\operatorname{div} \boldsymbol{z}^{\Omega} & =0 & & \text { on } \Omega, \\
\boldsymbol{z}^{\Omega} \times \boldsymbol{n} & =0 & & \text { on } \partial \Omega .
\end{aligned}
$$

The last equality holds because $\gamma_{t}(\boldsymbol{z})=0$, and the second, $\operatorname{div} \boldsymbol{z}^{\Omega}=0$, follows from (3.11). Now using the continuous imbedding of $\boldsymbol{H}_{0}(\operatorname{curl}, \Omega) \cap \boldsymbol{H}(\operatorname{div}, \Omega)$ into $\boldsymbol{H}^{1}(\Omega)$ on convex domains [20, we obtain

$$
\left\|\boldsymbol{z}^{\Omega}\right\|_{\boldsymbol{H}^{1}(\Omega)}=\left\|\operatorname{curl} \boldsymbol{p}_{\theta}\right\|_{\boldsymbol{H}^{1}(\Omega)} \leq C\|f\|_{r} .
$$

We will now use a result of [3, Proposition 3.17] that states that the $r, \theta$, and $z$ components of $\breve{H}^{1}(\Omega)$ are isometrically equivalent (up to a factor of $\sqrt{2 \pi}$ ) to 
$\widetilde{H}_{r}^{1}(D), \widetilde{H}_{r}^{1}(D)$, and $H_{r}^{1}(D)$, respectively. The first inequality of the theorem follows immediately from this and the above estimate, since $\|\boldsymbol{z}\|_{H_{r}^{1}(D)^{2}} \leq C\left\|\boldsymbol{z}^{\Omega}\right\|_{\boldsymbol{H}^{1}(\Omega)}$.

For the second estimate of the theorem, we again use the above-mentioned result of [3] to get

$$
\begin{aligned}
& \frac{1}{2 \pi}\left(\left\|\operatorname{curl} \boldsymbol{p}_{\theta}\right\|_{\boldsymbol{H}^{1}(\Omega)}^{2}+\left\|\boldsymbol{p}_{\theta}\right\|_{\boldsymbol{H}^{1}(\Omega)}^{2}\right) \\
& \quad=\frac{1}{2 \pi}\left(\left\|\left(-\partial_{z} p^{\Omega}\right) \boldsymbol{e}_{r}+\frac{1}{r} \partial_{r}\left(r p^{\Omega}\right) \boldsymbol{e}_{z}\right\|_{\boldsymbol{H}^{1}(\Omega)}^{2}+\left\|\boldsymbol{p}_{\theta}\right\|_{\boldsymbol{H}^{1}(\Omega)}^{2}\right) \\
& \quad=\left\|\partial_{z} p\right\|_{\widetilde{H}_{r}^{1}(D)}^{2}+\left\|\frac{1}{r} \partial_{r}(p r)\right\|_{H_{r}^{1}(D)}^{2}+\|p\|_{\widetilde{H}_{r}^{1}(D)}^{2} \\
& \quad \geq\|p\|_{\widetilde{H}_{r}^{2}(D)}^{2} .
\end{aligned}
$$

This together with (3.12) and (3.13) completes the proof.

Let us now consider the mixed finite element approximation of (3.1). The discrete problem is to find $\boldsymbol{z}_{h}$ in $\boldsymbol{W}_{h, \diamond}$ and $p_{h}$ in $S_{h}$ satisfying

$$
\begin{aligned}
\left(\boldsymbol{z}_{h}, \boldsymbol{w}_{h}\right)_{r}-\left(p_{h}, \operatorname{curl}_{r z} \boldsymbol{w}_{h}\right)_{r} & =0, & & \text { for all } \boldsymbol{w}_{h} \text { in } \boldsymbol{W}_{h, \diamond}, \\
\left(s_{h}, \operatorname{curl}_{r z} \boldsymbol{z}_{h}\right)_{r} & =\left(s_{h}, f\right)_{r}, & & \text { for all } s_{h} \text { in } S_{h} .
\end{aligned}
$$

At this point, we can proceed to analyze the discrete mixed method by verifying the conditions of the Babuška-Brezzi theory, which would yield a priori error estimates. However, for our multigrid analysis, we will need error estimates in a slightly more specialized form, so we will provide a direct error analysis. We will also prove a higher-order estimate obtained via duality. These results are collected in the next theorem.

Theorem 3.3. Suppose $\boldsymbol{z}$ in $\boldsymbol{H}_{r, \diamond}(\operatorname{curl}, D)$ and $p$ in $L_{r}^{2}(D)$ solve (3.1).

(1) There is a unique $\boldsymbol{z}_{h}$ in $\boldsymbol{W}_{h, \diamond}$ and a unique $p_{h}$ in $S_{h}$ satisfying (3.14).

(2) The following error estimate holds:

$$
\left\|\boldsymbol{z}-\boldsymbol{z}_{h}\right\|_{r} \leq\left\|\boldsymbol{z}-\Pi_{h}^{W} \boldsymbol{z}\right\|_{r} .
$$

(3) If $\Omega$ is convex, and $f$ is in $S_{h}$, then

$$
\left\|\Pi_{h}^{S} p-p_{h}\right\|_{r} \leq C h^{2}\|f\|_{r} .
$$

Proof. Proof of (11): Suppose $f=0$ in (3.14). Then by setting $\boldsymbol{w}_{h}=\boldsymbol{z}_{h}$, we get $z_{h}=0$. Then

$$
\left(p_{h}, \operatorname{curl}_{r z} \boldsymbol{w}_{h}\right)_{r}=0 \text { for all } \boldsymbol{w}_{h} \text { in } \boldsymbol{W}_{h, \diamond}
$$

which implies that $p_{h}=0$ by the exactness of (2.3), and this completes the proof.

Proof of (21): By subtracting (3.14) from (3.1), we get

$$
\begin{aligned}
\left(\boldsymbol{z}-\boldsymbol{z}_{h}, \boldsymbol{w}_{h}\right)-\left(p-p_{h}, \operatorname{curl}_{r z} \boldsymbol{w}_{h}\right)_{r}=0 & \text { for all } \boldsymbol{w}_{h} \text { in } \boldsymbol{W}_{h, \diamond}, \\
\left(s_{h}, \operatorname{curl}_{r z}\left(\boldsymbol{z}-\boldsymbol{z}_{h}\right)\right)_{r}=0 & \text { for all } s_{h} \text { in } S_{h} .
\end{aligned}
$$

Let $\boldsymbol{w}_{h}=\boldsymbol{z}_{h}-\Pi_{h}^{W} \boldsymbol{z}$. Then Theorem 2.1(2) implies that

$$
\begin{aligned}
\operatorname{curl}_{r z} \boldsymbol{w}_{h} & =\operatorname{curl}_{r z} \boldsymbol{z}_{h}-\operatorname{curl}_{r z}\left(\Pi_{h}^{W} \boldsymbol{z}\right)=\operatorname{curl}_{r z} \boldsymbol{z}_{h}-\Pi_{h}^{S}\left(\operatorname{curl}_{r z} \boldsymbol{z}\right) \\
& =\Pi_{h}^{S}\left(\operatorname{curl}_{r z}\left(\boldsymbol{z}_{h}-\boldsymbol{z}\right)\right) \\
& =0
\end{aligned}
$$


The last equality was due to (3.16). With this $\boldsymbol{w}_{h}$ in (3.15), we have

$$
\left(\boldsymbol{z}-\boldsymbol{z}_{h}, \boldsymbol{w}_{h}\right)_{r}=0 .
$$

In other words,

$$
\left(\boldsymbol{z}-\boldsymbol{z}_{h},\left(\boldsymbol{z}_{h}-\boldsymbol{z}\right)+\left(\boldsymbol{z}-\Pi_{h}^{W} \boldsymbol{z}\right)\right)_{r}=0,
$$

so an application of the Cauchy-Schwarz inequality shows that $\left\|\boldsymbol{z}-\boldsymbol{z}_{h}\right\|_{r}$ $\leq\left\|\boldsymbol{z}-\Pi_{h}^{W} \boldsymbol{z}\right\|_{r}$.

Proof of (3): We proceed by a duality argument, suitably modified. Let $\varepsilon_{z}$ in $\boldsymbol{H}_{r, \diamond}(\operatorname{curl}, D)$ and $\varepsilon_{p}$ in $L_{r}^{2}(D)$ solve (3.1) with $f$ set to $\Pi_{h}^{S} p-p_{h}$. Let their discrete counterparts be $\boldsymbol{\varepsilon}_{z, h}$ in $\boldsymbol{W}_{h, \diamond}$ and $\varepsilon_{p, h}$ in $S_{h}$, which solve (3.14) with $f$ set to $\Pi_{h}^{S} p-p_{h}$. Then by (3.14) and (3.15),

$$
\begin{aligned}
\left\|\Pi_{h}^{S} p-p_{h}\right\|_{r}^{2} & =\left(\Pi_{h}^{S} p-p_{h}, \operatorname{curl}_{r z} \boldsymbol{\varepsilon}_{z, h}\right)_{r} \\
& =\left(p-p_{h}, \operatorname{curl}_{r z} \boldsymbol{\varepsilon}_{z, h}\right)_{r} \\
& =\left(\boldsymbol{z}-\boldsymbol{z}_{h}, \boldsymbol{\varepsilon}_{z, h}\right)_{r} \\
& =\left(\boldsymbol{z}-\boldsymbol{z}_{h}, \boldsymbol{\varepsilon}_{z, h}-\boldsymbol{\varepsilon}_{z}\right)_{r}+\left(\boldsymbol{z}-\boldsymbol{z}_{h}, \boldsymbol{\varepsilon}_{z}\right)_{r} .
\end{aligned}
$$

Now, since $f$ is given to be in $S_{h}$,

$$
\operatorname{curl}_{r z} z=\operatorname{curl}_{r z} z_{h}=f .
$$

This together with the definition of $\left\{\varepsilon_{z}, \varepsilon_{p}\right\}$ implies that the last term in (3.17) vanishes:

$$
\left(\varepsilon_{z}, \boldsymbol{z}-\boldsymbol{z}_{h}\right)_{r}=\left(\varepsilon_{p}, \operatorname{curl}_{r z}\left(\boldsymbol{z}-\boldsymbol{z}_{h}\right)\right)=0 .
$$

Using this in (3.17) and continuing,

$$
\begin{array}{rlrl}
\left\|\Pi_{h}^{S} p-p_{h}\right\|_{r}^{2} & =\left(\boldsymbol{z}-\boldsymbol{z}_{h}, \boldsymbol{\varepsilon}_{z, h}-\boldsymbol{\varepsilon}_{z}\right)_{r} & & \\
& \leq\left\|\boldsymbol{z}-\boldsymbol{z}_{h}\right\|_{r}\left\|\varepsilon_{z, h}-\boldsymbol{\varepsilon}_{z}\right\|_{r} & \\
& \leq\left\|\boldsymbol{z}-\Pi_{h}^{W} \boldsymbol{z}\right\|_{r}\left\|\varepsilon_{z}-\Pi_{h}^{W} \varepsilon_{z}\right\|_{r} & & \text { by item (2) } \\
& \leq C h^{2}|\boldsymbol{z}|_{H_{r}^{1}(D)^{2}}\left|\varepsilon_{z}\right|_{H_{r}^{1}(D)^{2}} & & \text { by Corollary 2.1 }
\end{array}
$$

Since the revolution of $D$ is convex, by the regularity result of Theorem 3.2 .

$$
\left|\varepsilon_{z}\right|_{H_{r}^{1}(D)^{2}} \leq C\left\|\Pi_{h}^{S} p-p_{h}\right\|_{r} .
$$

Thus,

$$
\left\|\Pi_{h}^{S} p-p_{h}\right\|_{r}^{2} \leq C h^{2}|\boldsymbol{z}|_{H_{r}^{1}(D)}\left\|\Pi_{h}^{S} p-p_{h}\right\|_{r} .
$$

Canceling the common factor and applying Theorem 3.2 again, we obtain the required estimate.

Remark 3.1. Item (3) of Theorem 3.3 can be thought of as a superconvergence result, as it shows that we obtain quadratic convergence for $\Pi_{h}^{S} p-p_{h}$ even when using piecewise constant approximation spaces. In this respect, this result is similar to certain known superconvergence error estimates derived via duality arguments for the Raviart-Thomas mixed method [16, 19, 27, albeit without a degenerate weight function. 
Remark 3.2. There is an analogue of the mixed problem (3.1) in the case of the fully three-dimensional curl curl operator, sometimes called the dual mixed formulation (see e.g. [7, where it is used for eigenvalue analysis). However, this method is not practically popular in the 3D case as its implementation requires a basis for exactly divergence-free finite element spaces, which is not easy to construct. This difficulty is absent in the axisymmetric case.

\section{The MUltigrid ALGORITHM}

In this section, we present the multigrid algorithm and state a uniform convergence result for the algorithm. We now assume the typical geometrical multigrid setting, where the discrete solution space is based on the finest mesh $\mathscr{T}$ in a sequence of nested refinements of a coarse mesh. Let $\mathscr{T}_{1}$ be the coarsest mesh subdividing $D$. Typically $\mathscr{T}_{1}$ is small enough so that the cost of solving our finite element problem on it is negligible. For $k=2,3, \ldots, J$, the mesh $\mathscr{T}_{k}$ is obtained from $\mathscr{T}_{k-1}$ by connecting the midpoints of all edges. We want to efficiently solve a finite element problem on the mesh $\mathscr{T}=\mathscr{T}_{J}$ by a multigrid method.

The multilevel finite element spaces are Nédélec spaces on each of the meshes; i.e., let

$$
\boldsymbol{W}_{k}=\left\{\boldsymbol{v} \in \boldsymbol{H}_{r}(\operatorname{curl}, D):\left.\boldsymbol{v}\right|_{K} \in \boldsymbol{N}_{1} \text { for all } K \in \mathscr{T}_{k}, \text { and } \gamma_{t}(\boldsymbol{v})=0\right\} .
$$

Define $\Lambda_{k}: \boldsymbol{W}_{k} \rightarrow \boldsymbol{W}_{k}$ by

$$
\left(\Lambda_{k} \boldsymbol{u}_{k}, \boldsymbol{v}_{k}\right)_{r}=\Lambda\left(\boldsymbol{u}_{k}, \boldsymbol{v}_{k}\right) \quad \text { for all } \boldsymbol{u}_{k}, \boldsymbol{v}_{k} \in \boldsymbol{W}_{k} .
$$

The multigrid algorithm we present is for solving a linear system on the finest level, of the type $\Lambda_{J} \boldsymbol{u}=\boldsymbol{f}$.

To describe the algorithm, we first need to define certain smoothing operators $R_{k}: \boldsymbol{W}_{k} \mapsto \boldsymbol{W}_{k}$. These could be additive or multiplicative subspace correction operators based on any of the subspace decompositions of [2] and [22]. To describe them, first let $D_{k}^{\mathfrak{v}}$ denote the "vertex patch" domain formed by the union of all triangles in $\mathscr{T}_{k}$ connected to the mesh vertex $\mathfrak{v}$. Define $\boldsymbol{W}_{k}^{\mathfrak{v}}=\left\{\boldsymbol{v} \in \boldsymbol{W}_{k}: \operatorname{supp}(\boldsymbol{v}) \subseteq\right.$ $\left.D_{k}^{\mathfrak{p}}\right\}$. For every mesh edge $\mathfrak{e}$, let $\Phi_{\mathfrak{e}}$ denote the Whitney edge basis function, and let $\boldsymbol{W}_{k}^{\mathfrak{e}}$ denote $\operatorname{span}\left(\Phi_{\mathfrak{e}}\right)$. The decomposition of [2], adapted to our setting, is

$$
\boldsymbol{W}_{k}=\sum_{\mathfrak{v} \in \mathscr{Y}_{k}} \boldsymbol{W}_{k}^{\mathfrak{v}}+\sum_{\mathfrak{e} \in \mathscr{E}_{k}^{*}} \boldsymbol{W}_{k}^{\mathfrak{e}}
$$

where $\mathscr{V}_{k}$ is the set of nodes in the mesh $\mathscr{T}_{k}$ that are not on $\bar{\Gamma}_{1}$, and $\mathscr{E}_{k}^{*}$ is the set of edges of the mesh $\mathscr{T}_{k}$ that are not on $\Gamma_{1}$ but have both nodes on $\Gamma_{1}$. The last set $\mathscr{E}_{k}^{*}$ may appear "pathological," but it is needed in mixed boundary condition cases such as ours, as its edges are not covered by any of the vertex patches in the first sum of (4.1).

To be clear, let us exhibit the decomposition for a $\boldsymbol{u}_{k}$ in $\boldsymbol{W}_{k}$. Let $\mathscr{E}_{k}$ denote the set of edges in the mesh that are not on $\Gamma_{1}$. Then the basis expansion of $\boldsymbol{u}_{k}$ is

$$
\boldsymbol{u}_{k}=\sum_{\mathfrak{e} \in \mathscr{E}_{k}} c_{\mathfrak{e}} \Phi_{\mathfrak{e}}
$$

Now, for each $\mathfrak{v} \in \mathscr{V}_{k}$, define $\mathscr{E}_{k, \mathfrak{v}}^{1}$ and $\mathscr{E}_{k, \mathfrak{v}}^{2}$ as

$$
\begin{aligned}
& \mathscr{E}_{k, \mathfrak{v}}^{1}=\left\{\mathfrak{e} \in \mathscr{E}_{k}: \text { one endpoint of } \mathfrak{e} \text { is } \mathfrak{v} \text { and the other is on } \bar{\Gamma}_{1}\right\}, \\
& \mathscr{E}_{k, \mathfrak{v}}^{2}=\left\{\mathfrak{e} \in \mathscr{E}_{k}: \text { one endpoint of } \mathfrak{e} \text { is } \mathfrak{v} \text { and the other is not on } \bar{\Gamma}_{1}\right\} .
\end{aligned}
$$


When summing over the vertex patches, the edges of $\mathscr{E}_{k, \mathfrak{v}}^{2}$ are counted twice. Hence, setting

$$
\boldsymbol{u}_{k}^{\mathfrak{v}}:=\sum_{\mathfrak{e} \in \mathscr{E}_{k, \mathfrak{v}}} c_{\mathfrak{e}} \Phi_{\mathfrak{e}}+\sum_{\mathfrak{e} \in \mathscr{E}_{k, \mathfrak{v}}} \frac{1}{2} c_{\mathfrak{e}} \Phi_{\mathfrak{e}}
$$

we have

$$
\boldsymbol{u}_{k}=\sum_{\mathfrak{v} \in \mathscr{V}_{k}} \boldsymbol{u}_{k}^{\mathfrak{v}}+\sum_{\mathfrak{e} \in \mathscr{E}_{k}^{*}} c_{\mathfrak{e}} \Phi_{\mathfrak{e}} .
$$

This shows that $\boldsymbol{W}_{k}$ can indeed be decomposed as in (4.1).

The other subspace decomposition, due to [22], reads as follows in our application:

$$
\boldsymbol{W}_{k}=\sum_{\mathfrak{e} \in \mathscr{E}_{k}} \boldsymbol{W}_{k}^{\mathfrak{e}}+\sum_{\mathfrak{v} \in \mathscr{V}_{k}} \operatorname{grad}_{r z} V_{k}^{\mathfrak{v}},
$$

where $V_{k}^{\mathfrak{v}}$ is the (one-dimensional) space of continuous scalar functions supported on $D_{k}^{\mathfrak{v}}$ which are linear on each triangle of $D_{k}^{\mathfrak{v}}$ and vanish on $\partial D_{k}^{\mathfrak{v}}$.

We can use either (4.1) or (4.2) to construct additive or multiplicative smoothers. The details are standard, so we present only the algorithm for the block Gauss-Seidel type multiplicative smoothing iteration

$$
\boldsymbol{u}_{i+1}=\mathrm{GS}_{k}\left(\boldsymbol{u}_{i}, \boldsymbol{f}\right)
$$

where the procedure $\operatorname{GS}_{k}(\cdot, \cdot)$ is given below. Let $\boldsymbol{W}_{k, i}, i=1,2, \ldots, N_{k}$, be an enumeration of the subspaces in either of the decompositions (4.1) or (4.2). Define $\Lambda_{k, i}: \boldsymbol{W}_{k, i} \mapsto \boldsymbol{W}_{k, i}$ by

$$
\left(\Lambda_{k, i} \boldsymbol{v}_{i}, \boldsymbol{w}_{i}\right)_{r}=\Lambda\left(\boldsymbol{v}_{i}, \boldsymbol{w}_{i}\right), \quad \text { for all } \boldsymbol{v}_{i}, \boldsymbol{w}_{i} \text { in } \boldsymbol{W}_{k, i} .
$$

Let the $L_{r}^{2}(D)^{2}$-orthogonal projection onto $\boldsymbol{W}_{k, i}$ be denoted by $Q_{k, i}$.

Algorithm 4.1 (Multiplicative smoothing). Given $\boldsymbol{u}_{i}$ in $\boldsymbol{W}_{k}$, calculate $\boldsymbol{u}_{i+1}=$ $\mathrm{GS}_{k}\left(\boldsymbol{u}_{i}, \boldsymbol{f}\right)$ in $\boldsymbol{W}_{k}$ as follows:

(1) Set $\boldsymbol{u}_{i}^{(0)}=\boldsymbol{u}_{i}$.

(2) For $j=1,2, \ldots, N_{k}$, compute

$$
\boldsymbol{u}_{i}^{(j)}=\boldsymbol{u}_{i}^{(j-1)}+\Lambda_{k, j}^{-1} Q_{k, j}\left(\boldsymbol{f}-\Lambda_{k} \boldsymbol{u}_{i}^{(j-1)}\right) .
$$

(3) Set the result $\boldsymbol{u}_{i+1}$ to be $\boldsymbol{u}_{i}^{\left(N_{k}\right)}$.

Standard arguments show that this iteration can be rewritten as

$$
\boldsymbol{u}_{i+1}=\boldsymbol{u}_{i}+R_{k}\left(\boldsymbol{f}-\Lambda_{k} \boldsymbol{u}_{i}\right)
$$

with

$$
R_{k}=\left(I-\left(I-P_{k, N_{k}}\right)\left(I-P_{k, N_{k}-1}\right) \cdots\left(I-P_{k, 1}\right)\right) \Lambda_{k}^{-1},
$$

where $P_{k, j}$ is the orthogonal projection into $\boldsymbol{W}_{k, j}$ in the $\Lambda(\cdot, \cdot)$-inner product. With such a smoother $R_{k}$, or an additive Jacobi type smoother based on the same decompositions (whose details we omit), we can now describe the multigrid algorithm. Let the $L_{r}^{2}(D)^{2}$-orthogonal projection onto $\boldsymbol{W}_{k}$ be denoted by $Q_{k}$.

Algorithm 4.2 (V-cycle). Given $\boldsymbol{u}$ and $\boldsymbol{f}$ in $\boldsymbol{W}_{k}$, define the output $\mathrm{MG}_{k}(\boldsymbol{u}, \boldsymbol{f})$ in $\boldsymbol{W}_{k}$ by the following recursive procedure:

(1) Set $\mathrm{MG}_{1}(\boldsymbol{u}, \boldsymbol{f})=\Lambda_{1}^{-1} \boldsymbol{f}$.

(2) For $k>1$, define $\mathrm{MG}_{k}(\boldsymbol{u}, \boldsymbol{f})$ recursively:

(a) $\boldsymbol{v}^{(1)}=\boldsymbol{u}+R_{k}\left(\boldsymbol{f}-\Lambda_{k} \boldsymbol{u}\right)$. 
(b) $\boldsymbol{v}^{(2)}=\boldsymbol{v}^{(1)}+\mathrm{MG}_{k-1}\left(\mathbf{0}, Q_{k-1}\left(\boldsymbol{f}-\Lambda_{k} \boldsymbol{v}^{(1)}\right)\right)$.

(c) $\boldsymbol{v}^{(3)}=\boldsymbol{v}^{(2)}+R_{k}^{t}\left(\boldsymbol{f}-\Lambda_{k} \boldsymbol{v}^{(2)}\right)$.

(d) $\operatorname{Set} \mathrm{MG}_{k}(\boldsymbol{u}, \boldsymbol{f})=\boldsymbol{v}^{(3)}$.

It is well known [10] that the $\mathrm{V}$-cycle iterates $\boldsymbol{x}_{i+1}=\mathrm{MG}_{k}\left(\boldsymbol{x}_{i}, \boldsymbol{f}\right)$, approximating the exact solution $\boldsymbol{x}=\Lambda_{k}^{-1} \boldsymbol{f}$, are connected through a linear error reduction operator $\mathcal{E}_{k}$, i.e.,

$$
\boldsymbol{x}-\boldsymbol{x}_{i+1}=\mathcal{E}_{k}\left(\boldsymbol{x}-\boldsymbol{x}_{i}\right) .
$$

The following is our main result on the convergence of the V-cycle algorithm. Its proof is given in the next section.

Theorem 4.1. Assume that $\Omega$, the revolution of $D$, is convex. There exists a positive number $\delta<1$ such that

$$
0 \leq \Lambda\left(\mathcal{E}_{k} \boldsymbol{u}, \boldsymbol{u}\right) \leq \delta \Lambda(\boldsymbol{u}, \boldsymbol{u}), \quad \text { for all } \boldsymbol{u} \text { in } \boldsymbol{W}_{k} \text { and all } k \geq 1 .
$$

The number $\delta$ is independent of the mesh size and refinement level.

\section{MULTIGRID ANALYSIS}

In this section, we prove Theorem 4.1 by verifying two conditions in a standard abstract framework for multigrid analysis [2, 9, 10, 12. We state the conditions and its implications as the next lemma and omit its well-known proof. The analysis of this section is heavily based on the techniques introduced in [2]. Let $P_{k}$ denote the orthogonal projection into $\boldsymbol{W}_{k}$ in the $\Lambda(\cdot, \cdot)$-inner product.

Lemma 5.1. The assertion of Theorem 4.1 follows from the two conditions below:

(1) Existence of a stable decomposition: There exists a constant $C_{1}>0$ independent of the meshsizes and $k$, such that for all $\boldsymbol{v}$ in $\left(I-P_{k-1}\right) \boldsymbol{W}_{k}$, there is a decomposition

$$
\boldsymbol{v}=\sum_{j=1}^{N_{k}} \boldsymbol{v}_{j}, \quad \text { with } \boldsymbol{v}_{j} \text { in } \boldsymbol{W}_{k, j}
$$

satisfying

$$
\sum_{j=1}^{N_{k}} \Lambda\left(\boldsymbol{v}_{j}, \boldsymbol{v}_{j}\right) \leq C_{1} \Lambda(\boldsymbol{v}, \boldsymbol{v})
$$

(2) Limited interaction: There exists a constant $C_{2}>0$, independent of $k$, such that

$$
\sum_{j=1}^{N_{k}} \sum_{l=1}^{N_{k}}\left|\Lambda\left(\boldsymbol{v}_{j}, \boldsymbol{w}_{l}\right)\right| \leq C_{2}\left(\sum_{j=1}^{N_{k}} \Lambda\left(\boldsymbol{v}_{j}, \boldsymbol{v}_{j}\right)\right)^{\frac{1}{2}}\left(\sum_{k=1}^{N_{k}} \Lambda\left(\boldsymbol{w}_{l}, \boldsymbol{w}_{l}\right)\right)^{\frac{1}{2}}
$$

for all $\boldsymbol{v}_{j}$ in $\boldsymbol{W}_{k, j}, \boldsymbol{w}_{l}$ in $\boldsymbol{W}_{k, l}$, and $k \geq 1$.

The remainder of this section is devoted to the verification of the two conditions of Lemma 5.1. Note that the first condition only involves functions on two levels, $k$ and $k-1$. The second involves an inequality of functions in just one level $k$. For this reason, we can simplify our notation and use subscripts $H$ and $h$ for $k-1$ and $k$, respectively. The mesh $\mathscr{T}_{h}$ is a refinement of $\mathscr{T}_{H}$, and $H=2 h$. Previously defined notation with these new subscripts have the obvious definitions; e.g., $\Pi_{H}^{S}$ denotes the weighted $L_{r}^{2}(D)$-orthogonal projection into $S_{H}$, the space of piecewise constant 
functions with respect to the mesh $\mathscr{T}_{H}$, etc. Before verifying the conditions, we need a number of preliminary results. Throughout this section we tacitly assume that $\Omega$, the revolution of $D$, is convex in order to invoke the regularity results of Theorem 3.2 .

Lemma 5.2. For all $p_{h}$ in $S_{h}$,

$$
\left\|p_{h}-\Pi_{H}^{S} p_{h}\right\|_{r} \leq C H\left\|\operatorname{curl}_{r z}^{\prime} p_{h}\right\|_{r} .
$$

Proof. Given $p_{h}$ in $S_{h}$, define $\boldsymbol{z}$ in $\boldsymbol{H}_{r, \diamond}(\operatorname{curl}, D)$ and $p$ in $L_{r}^{2}(D)$ as the solution of (3.1) with $f=\operatorname{curl}_{r z} \operatorname{curl}_{r z}^{\prime} p_{h}$, i.e.,

$$
\begin{aligned}
(\boldsymbol{z}, \boldsymbol{w})_{r}-\left(p, \operatorname{curl}_{r z} \boldsymbol{w}\right)_{r} & =0 & & \text { for all } \boldsymbol{w} \text { in } \boldsymbol{H}_{r, \diamond}(\operatorname{curl}, D), \\
\left(s, \operatorname{curl}_{r z} \boldsymbol{z}\right)_{r} & =\left(s, \operatorname{curl}_{r z} \operatorname{curl}_{r z}^{\prime} p_{h}\right)_{r} & & \text { for all } s \text { in } L_{r}^{2}(D) .
\end{aligned}
$$

Then, with $\boldsymbol{z}_{h}=\operatorname{curl}_{r z}^{\prime} p_{h}$, the pair $\left\{\boldsymbol{z}_{h}, p_{h}\right\}$ obviously satisfies

$$
\begin{aligned}
\left(\boldsymbol{z}_{h}, \boldsymbol{w}_{h}\right)_{r}-\left(p_{h}, \operatorname{curl}_{r z} \boldsymbol{w}_{h}\right)_{r} & =0 & & \text { for all } \boldsymbol{w}_{h} \text { in } \boldsymbol{W}_{h, \diamond,}, \\
\left(s_{h}, \operatorname{curl}_{r z} \boldsymbol{z}_{h}\right)_{r} & =\left(s_{h}, \operatorname{curl}_{r z} \operatorname{curl}_{r z}^{\prime} p_{h}\right)_{r} & & \text { for all } s_{h} \text { in } S_{h} .
\end{aligned}
$$

Moreover,

$$
\operatorname{curl}_{r z} \boldsymbol{z}=\operatorname{curl}_{r z} \boldsymbol{z}_{h} \quad \text { in } L_{r}^{2}(D)
$$

By the triangle inequality,

$$
\left\|p_{h}-\Pi_{H}^{S} p_{h}\right\|_{r} \leq\left\|p_{h}-p\right\|_{r}+\left\|p-\Pi_{H}^{S} p\right\|_{r}+\left\|\Pi_{H}^{S} p-\Pi_{H}^{S} p_{h}\right\|_{r} .
$$

We now estimate each of the terms on the right hand side above.

Beginning with the middle term, and using a standard weighted norm approximation estimate (see e.g. 4, 11]), we have

$$
\left\|p-\Pi_{H}^{S} p\right\|_{r}^{2} \leq C H^{2}|p|_{H_{r}^{1}(D)}^{2} \leq C H^{2}\left(\|p\|_{H_{r}^{1}(D)}^{2}+\left\|r^{-1} p\right\|_{r}^{2}\right) .
$$

The right hand side is bounded because $p$ is in $\widetilde{H}_{r}^{2}(D)$, by Theorem 3.2 . Moreover, as in [21, Proposition 3.1], it can be bounded further by $C H^{2}\left(\left\|r^{-1} \partial_{r}(r p)\right\|_{r}^{2}+\right.$ $\left.\left\|\partial_{z} p\right\|_{r}^{2}\right)$, which is the same as $C H^{2}\left\|\operatorname{curl}_{r z} p\right\|_{r}^{2}$. Thus,

$$
\left\|p-\Pi_{H}^{S} p\right\|_{r} \leq C H\left\|\operatorname{curl}_{r z} p\right\|_{r} .
$$

Furthermore, since

$$
\begin{aligned}
\left\|\operatorname{curl}_{r z} p\right\|_{r}^{2} & =\left(\operatorname{curl}_{r z} p, \boldsymbol{z}\right)_{r} & & \text { cf. }(\underline{3.10}) \\
& =\left(p, \operatorname{curl}_{r z} \boldsymbol{z}\right)_{r} & & \text { by (1.6) } \\
& =\left(p, \operatorname{curl}_{r z} \boldsymbol{z}_{h}\right)_{r} & & \text { by (5.2) } \\
& =\left(\operatorname{curl}_{r z} p, \boldsymbol{z}_{h}\right)_{r} & & \text { by (1.6) } \\
& =\left(\operatorname{curl}_{r z} p, \operatorname{curl}_{r z}^{\prime} p_{h}\right)_{r}, & &
\end{aligned}
$$

by the Cauchy-Schwarz inequality, we obtain $\left\|\operatorname{curl}_{r z} p\right\|_{r} \leq\left\|\operatorname{curl}_{r z}^{\prime} p_{h}\right\|_{r}$. Thus (5.4) yields

$$
\left\|p-\Pi_{H}^{S} p\right\|_{r} \leq C H\left\|\operatorname{curl}_{r z}^{\prime} p_{h}\right\|_{r} .
$$

Next, consider the first term on the right hand side of (5.3). Using the triangle inequality,

$$
\left\|p-p_{h}\right\|_{r} \leq\left\|p-\Pi_{h}^{S} p\right\|_{r}+\left\|\Pi_{h}^{S} p-p_{h}\right\|_{r}
$$


The term $\left\|p-\Pi_{h}^{S} p\right\|_{r}$ can be bounded by the same type of argument that led to (5.5). Theorem 3.3(3) provides a bound for the other term. Then the inverse estimate [4, Lemma 4] yields

$$
\begin{aligned}
\left\|p-p_{h}\right\|_{r} & \leq C h\left\|\operatorname{curl}_{r z}^{\prime} p_{h}\right\|_{r}+C h^{2}\left\|\operatorname{curl}_{r z} \operatorname{curl}_{r z}^{\prime} p_{h}\right\|_{r} \\
& \leq C h\left\|\operatorname{curl}_{r z}^{\prime} p_{h}\right\|_{r} .
\end{aligned}
$$

The only remaining term on the right hand side of (5.3) is bounded by using (5.6) and the fact that orthogonal projectors have unit norm:

$$
\left\|\Pi_{H}^{S} p-\Pi_{H}^{S} p_{h}\right\|_{r} \leq\left\|p-p_{h}\right\|_{r} \leq C h\left\|\operatorname{curl}_{r z}^{\prime} p_{h}\right\|_{r} .
$$

Collecting the estimates of (5.5), (5.6) and (5.7) in (5.3), we have

$$
\left\|p_{h}-\Pi_{H}^{S} p_{h}\right\|_{r} \leq C(H+h)\left\|\operatorname{curl}_{r z}^{\prime} p_{h}\right\|_{r} .
$$

Since $h \leq C H$, this completes the proof.

The next lemma is crucial in proving the uniform convergence of the multigrid $\mathrm{V}$-cycle and is modeled after the lemmas in 2. We shall make significant use of the weighted discrete Helmholtz decomposition discussed in Section 2 Recall that as per our previous remarks on the notation, $P_{H}$ denotes the $\Lambda$-orthogonal projection into the coarser of the two spaces.

Lemma 5.3. Let $\boldsymbol{w}_{h}$ be in $\boldsymbol{W}_{h, \diamond}$. If the weighted discrete Helmholtz decomposition of $\boldsymbol{w}_{h}-P_{H} \boldsymbol{w}_{h}$ is

$$
\boldsymbol{w}_{h}-P_{H} \boldsymbol{w}_{h}=\operatorname{grad}_{r z} \phi_{h}+\operatorname{curl}_{r z}^{\prime} a_{h},
$$

with $\phi_{h}$ in $V_{h, \diamond}$ and $a_{h}$ in $S_{h}$, then

$$
\begin{aligned}
\left\|\phi_{h}\right\|_{r} & \leq C H\left\|\left(I-P_{H}\right) \boldsymbol{w}_{h}\right\|_{r} \\
\left\|\operatorname{curl}_{r z}^{\prime} a_{h}\right\|_{r} & \leq C H\left\|\left(I-P_{H}\right) \boldsymbol{w}_{h}\right\|_{\Lambda},
\end{aligned}
$$

where $\|\cdot\|_{\Lambda} \equiv\|\cdot\|_{r, \text { curl }}$.

Proof. We first prove the second inequality. Define $\boldsymbol{z}_{h}$ in $\boldsymbol{W}_{h, \diamond}$, given the above $a_{h}$, by

$$
\Lambda\left(\boldsymbol{z}_{h}, \boldsymbol{q}_{h}\right)=\left(\operatorname{curl}_{r z}^{\prime} a_{h}, \boldsymbol{q}_{h}\right)_{r} \quad \text { for all } \boldsymbol{q}_{h} \text { in } \boldsymbol{W}_{h, \diamond} .
$$

Observe that $\boldsymbol{z}_{h}$ is orthogonal to $\operatorname{grad}_{r z} V_{h, \diamond}$. By setting $\boldsymbol{q}_{h}=\operatorname{curl}_{r z}^{\prime} a_{h}$ above,

$$
\begin{aligned}
\left\|\operatorname{curl}_{r z}^{\prime} a_{h}\right\|_{r}^{2} & =\Lambda\left(\boldsymbol{z}_{h}, \operatorname{curl}_{r z}^{\prime} a_{h}\right)=\Lambda\left(\boldsymbol{z}_{h},\left(I-P_{H}\right) \boldsymbol{w}_{h}-\operatorname{grad}_{r z} \phi_{h}\right) \\
& =\Lambda\left(\boldsymbol{z}_{h},\left(I-P_{H}\right) \boldsymbol{w}_{h}\right)=\Lambda\left(\boldsymbol{z}_{h}-\boldsymbol{z}_{H},\left(I-P_{H}\right) \boldsymbol{w}_{h}\right) \\
& \leq\left\|\boldsymbol{z}_{h}-\boldsymbol{z}_{H}\right\|_{\Lambda}\left\|\left(I-P_{H}\right) \boldsymbol{w}_{h}\right\|_{\Lambda},
\end{aligned}
$$

for any $\boldsymbol{z}_{H}$ in $\boldsymbol{W}_{H, \diamond}$. Next, we choose a suitable $\boldsymbol{z}_{H}$ and estimate $\left\|\boldsymbol{z}_{h}-\boldsymbol{z}_{H}\right\|_{\Lambda}$.

To this end, first define $\boldsymbol{z}$ in $\boldsymbol{H}_{r, \diamond}(\operatorname{curl}, D)$, given the above $\boldsymbol{z}_{h}$, by (3.1) with $f=\operatorname{curl}_{r z} \boldsymbol{z}_{h}$, i.e.,

$$
\begin{aligned}
(\boldsymbol{z}, \boldsymbol{w})_{r}-\left(p, \operatorname{curl}_{r z} \boldsymbol{w}\right)_{r} & =0 & & \text { for all } \boldsymbol{w} \text { in } \boldsymbol{H}_{r, \diamond}(\operatorname{curl}, D), \\
\left(s, \operatorname{curl}_{r z} \boldsymbol{z}\right)_{r} & =\left(s, \operatorname{curl}_{r z} \boldsymbol{z}_{h}\right)_{r} & & \text { for all } s \text { in } L_{r}^{2}(D) .
\end{aligned}
$$

Then define $\boldsymbol{z}_{H}$ in $\boldsymbol{W}_{H, \diamond}$ by the analogue of (3.14) on the coarser of the meshes, i.e.,

$$
\begin{aligned}
\left(\boldsymbol{z}_{H}, \boldsymbol{w}_{H}\right)_{r}-\left(p_{H}, \operatorname{curl}_{r z} \boldsymbol{w}_{H}\right)_{r} & =0 & & \text { for all } \boldsymbol{w}_{H} \text { in } \boldsymbol{W}_{H, \diamond} \\
\left(s_{H}, \operatorname{curl}_{r z} \boldsymbol{z}_{H}\right)_{r} & =\left(s_{H}, \operatorname{curl}_{r z} \boldsymbol{z}_{h}\right)_{r} & & \text { for all } s_{H} \text { in } S_{H} .
\end{aligned}
$$


Since $\boldsymbol{z}_{h}$ is orthogonal to $\operatorname{grad}_{r z} V_{h, \diamond}$, it is clear that $\boldsymbol{z}_{h}$ is in the range of curl ${ }_{r z}^{\prime}$ by the weighted discrete Helmholtz decomposition of $\boldsymbol{W}_{h, \diamond}$. Thus, there is a unique $p_{h}$ in $S_{h}$ such that $\operatorname{curl}_{r z}^{\prime} p_{h}=\boldsymbol{z}_{h}$. In other words,

$$
\left(\boldsymbol{z}_{h}, \boldsymbol{w}_{h}\right)_{r}-\left(p_{h}, \operatorname{curl}_{r z} \boldsymbol{w}_{h}\right)_{r}=0 \quad \text { for all } \boldsymbol{w}_{h} \text { in } \boldsymbol{W}_{h, \diamond},
$$

which is the first equation of the formulation (3.14). The second equation of (3.14) is also satisfied by $\boldsymbol{z}_{h}$ trivially since $f=\operatorname{curl}_{r z} \boldsymbol{z}_{h}$. Therefore, by Theorem 3.3)(2),

$$
\begin{aligned}
\left\|\boldsymbol{z}-\boldsymbol{z}_{h}\right\|_{r} & \leq\left\|\boldsymbol{z}-\Pi_{h}^{W} \boldsymbol{z}\right\|_{r}, \\
\left\|\boldsymbol{z}-\boldsymbol{z}_{H}\right\|_{r} & \leq\left\|\boldsymbol{z}-\Pi_{H}^{W} \boldsymbol{z}\right\|_{r},
\end{aligned}
$$

which implies that

$$
\begin{aligned}
\left\|\boldsymbol{z}_{h}-\boldsymbol{z}_{H}\right\|_{r} & \leq\left\|\boldsymbol{z}-\Pi_{h}^{W} \boldsymbol{z}\right\|_{r}+\left\|\boldsymbol{z}-\Pi_{H}^{W} \boldsymbol{z}\right\|_{r} & & \text { by the triangle inequality, } \\
& \leq C H|\boldsymbol{z}|_{H_{r}^{1}(D)^{2}} & & \text { by Corollary 2.1. } \\
& \leq C H\left\|\operatorname{curl}_{r z} \boldsymbol{z}_{h}\right\|_{r} & & \text { by Theorem 3.2. }
\end{aligned}
$$

We also need to estimate $\left\|\operatorname{curl}_{r z}\left(\boldsymbol{z}_{h}-\boldsymbol{z}_{H}\right)\right\|_{r}$. By the definition of $\boldsymbol{z}_{H}$ and Lemma 5.2

$$
\begin{aligned}
\left\|\operatorname{curl}_{r z}\left(\boldsymbol{z}_{h}-\boldsymbol{z}_{H}\right)\right\|_{r} & =\left\|\operatorname{curl}_{r z} \boldsymbol{z}_{h}-\Pi_{H}^{S} \operatorname{curl}_{r z} \boldsymbol{z}_{h}\right\|_{r} \\
& \leq C H\left\|\operatorname{curl}_{r z}^{\prime} \operatorname{curl}_{r z} \boldsymbol{z}_{h}\right\|_{r} .
\end{aligned}
$$

Combining this with (5.10), we get

$$
\left\|\boldsymbol{z}_{h}-\boldsymbol{z}_{H}\right\|_{\Lambda}^{2} \leq C H^{2}\left(\left\|\operatorname{curl}_{r z} \boldsymbol{z}_{h}\right\|_{r}^{2}+\left\|\operatorname{curl}_{r z}^{\prime} \operatorname{curl}_{r z} \boldsymbol{z}_{h}\right\|_{r}^{2}\right) .
$$

This estimate is not yet in a form we can use in (5.9). To simplify its right hand side, define $\Lambda_{h}: \boldsymbol{W}_{h, \diamond} \rightarrow \boldsymbol{W}_{h, \diamond}$ by

$$
\left(\Lambda_{h} \boldsymbol{v}_{h}, \boldsymbol{w}_{h}\right)_{r}=\Lambda\left(\boldsymbol{v}_{h}, \boldsymbol{w}_{h}\right) \quad \text { for all } \boldsymbol{w}_{h} \text { in } \boldsymbol{W}_{h, \diamond} .
$$

We observe that $\Lambda_{h} z_{h}=\operatorname{curl}_{r z}^{\prime} a_{h}$ by (5.8), and

$$
\begin{aligned}
\left\|\Lambda_{h} \boldsymbol{z}_{h}\right\|_{r}^{2} & =\left(\Lambda_{h} \boldsymbol{z}_{h}, \Lambda_{h} \boldsymbol{z}_{h}\right)_{r}=\Lambda\left(\boldsymbol{z}_{h}, \Lambda_{h} \boldsymbol{z}_{h}\right) \\
& =\left(\boldsymbol{z}_{h}, \Lambda_{h} \boldsymbol{z}_{h}\right)_{r}+\left(\operatorname{curl}_{r z} \boldsymbol{z}_{h}, \operatorname{curl}_{r z}\left(\Lambda_{h} \boldsymbol{z}_{h}\right)\right)_{r} \\
& =\Lambda\left(\boldsymbol{z}_{h}, \boldsymbol{z}_{h}\right)+\Lambda\left(\operatorname{curl}_{r z}^{\prime} \operatorname{curl}_{r z} \boldsymbol{z}_{h}, \boldsymbol{z}_{h}\right) \\
& =\left\|\boldsymbol{z}_{h}\right\|_{r}^{2}+2\left\|\operatorname{curl}_{r z} \boldsymbol{z}_{h}\right\|_{r}^{2}+\left\|\operatorname{curl}_{r z}^{\prime} \operatorname{curl}_{r z} \boldsymbol{z}_{h}\right\|_{r}^{2} .
\end{aligned}
$$

Hence, returning to (5.11) and overestimating its right hand side,

$$
\left\|\boldsymbol{z}_{h}-\boldsymbol{z}_{H}\right\|_{\Lambda}^{2} \leq C H^{2}\left\|\Lambda_{h} \boldsymbol{z}_{h}\right\|_{r}^{2}=C H^{2}\left\|\operatorname{curl}_{r z}^{\prime} a_{h}\right\|_{r}^{2} .
$$

Using this estimate in (5.9), we have

$$
\left\|\operatorname{curl}_{r z}^{\prime} a_{h}\right\|_{r}^{2} \leq C H\left\|\operatorname{curl}_{r z}^{\prime} a_{h}\right\|_{r}\left\|\left(I-P_{H}\right) \boldsymbol{w}_{h}\right\|_{\Lambda},
$$

from which the second inequality of the lemma follows.

It now only remains to prove the first estimate of the lemma. Let $\psi$ in $H_{r, \diamond}^{1}(D)$ be the unique solution (see [21]) of

$$
\left(\operatorname{grad}_{r z} \psi, \operatorname{grad}_{r z} \eta\right)_{r}=\left(\phi_{h}, \eta\right)_{r} \quad \text { for all } \eta \text { in } H_{r, \diamond}^{1}(D) \text {. }
$$

Then [21, Theorem 2.1] gives the regularity estimate

$$
|\psi|_{H_{r}^{2}(D)} \leq C\left\|\phi_{h}\right\|_{r} .
$$


Observe that for any $\psi_{H}$ in $V_{H, \diamond}$

$$
\begin{aligned}
\left(\operatorname{grad}_{r z} \phi_{h}, \operatorname{grad}_{r z} \psi_{H}\right)_{r} & =\Lambda\left(\operatorname{grad}_{r z} \phi_{h}, \operatorname{grad}_{r z} \psi_{H}\right) \\
& =\Lambda\left(\boldsymbol{w}_{h}-P_{H} \boldsymbol{w}_{h}, \operatorname{grad}_{r z} \psi_{H}\right)=0 .
\end{aligned}
$$

We will use this with $\psi_{H}=\widehat{\Pi}_{H}^{V} \psi$, where $\widehat{\Pi}_{H}^{V}$ is the previously mentioned projection of [17. Proceeding by a standard duality argument 26,

$$
\begin{aligned}
\left\|\phi_{h}\right\|_{r}^{2} & =\left(\phi_{h}, \phi_{h}\right)_{r}=\left(\operatorname{grad}_{r z} \psi, \operatorname{grad}_{r z} \phi_{h}\right)_{r} & & \text { by (15.12) } \\
& =\left(\operatorname{grad}_{r z} \psi-\operatorname{grad}_{r z} \widehat{\Pi}_{H}^{V} \psi, \operatorname{grad}_{r z} \phi_{h}\right)_{r} & & \text { by (15.14) } \\
& \leq C H|\psi|_{H_{r}^{2}(D)}\left\|\operatorname{grad}_{r z} \phi_{h}\right\|_{r} & & \text { by [17, Lemma 5.3] } \\
& \leq C H\left\|\phi_{h}\right\|_{r}\left\|\operatorname{grad}_{r z} \phi_{h}\right\|_{r} & & \text { by (5.13). }
\end{aligned}
$$

Canceling the common factor, and using the stability estimate (2.4),

$$
\begin{aligned}
\left\|\phi_{h}\right\|_{r} & \leq C H\left\|\operatorname{grad}_{r z} \phi_{h}\right\|_{r} \\
& \leq C H\left\|\boldsymbol{w}_{h}-P_{H} \boldsymbol{w}_{h}\right\|_{r},
\end{aligned}
$$

which finishes the proof of the lemma.

We can now prove the convergence of the multigrid as an iterative method.

Proof of Theorem 4.1, By Lemma 5.1, we only need to verify the two conditions there. For verifying the second condition on the limited interaction of smoothing subspaces, we can use standard techniques [2, 12. Hence we omit it.

Let us now verify the first condition on the existence of a stable decomposition for the case of the smoothing subspaces of 2, namely (4.1). Given any $\boldsymbol{w}_{k}$ in $\left(I-P_{k-1}\right) \boldsymbol{W}_{k}$, let

$$
\boldsymbol{w}_{k}=\operatorname{grad}_{r z} \phi_{k}+\boldsymbol{r}_{k}
$$

be its weighted discrete Helmholtz decomposition, with $\phi_{k}$ in $V_{k}=\left\{v \in H_{r, \diamond}^{1}(D)\right.$ : $\left.v\right|_{K} \in P_{1}$ for all $\left.K \in \mathscr{T}_{k}\right\}$ and $\boldsymbol{r}_{k}$ in $\boldsymbol{W}_{k}$. By Lemma 5.3 .

$$
\begin{aligned}
\left\|\phi_{k}\right\|_{r} & \leq C h_{k-1}\left\|\boldsymbol{w}_{k}\right\|_{r}, \\
\left\|\boldsymbol{r}_{k}\right\|_{r} & \leq C h_{k-1}\left\|\boldsymbol{w}_{k}\right\|_{\Lambda} .
\end{aligned}
$$

Let $V_{k}^{\mathfrak{v}}=\left\{v \in V_{k}: \operatorname{supp}(v) \subseteq D_{k}^{\mathfrak{v}}\right\}$. Then, by using the decomposition

$$
V_{k}=\sum_{\mathfrak{v} \in \mathscr{V}_{k}} V_{k}^{\mathfrak{v}},
$$

we split

$$
\phi_{k}=\sum_{\mathfrak{v} \in \mathscr{Y}_{k}} \phi_{k}^{\mathfrak{v}}, \quad \text { with } \phi_{k}^{\mathfrak{v}} \text { in } V_{k}^{\mathfrak{v}},
$$

while we split $\boldsymbol{r}_{k}$ by the decomposition of (4.1) as

$$
\boldsymbol{r}_{k}=\sum_{\mathfrak{v} \in \mathscr{Y}_{k}} \boldsymbol{r}_{k}^{\mathfrak{v}}+\sum_{\mathfrak{e} \in \mathscr{E}_{k}^{*}} \boldsymbol{r}_{k}^{\mathfrak{e}}, \quad \text { with } \boldsymbol{r}_{k}^{\mathfrak{v}} \text { in } \boldsymbol{W}_{k}^{\mathfrak{v}}, \boldsymbol{r}_{k}^{\mathfrak{e}} \text { in } \boldsymbol{W}_{k}^{\mathfrak{e}}
$$

Setting $\boldsymbol{w}_{k}^{\mathfrak{v}}=\operatorname{grad}_{r z} \phi_{k}^{\mathfrak{v}}+\boldsymbol{r}_{k}^{\mathfrak{v}}$, we want to show that

$$
\sum_{\mathfrak{v} \in \mathscr{V}_{k}} \Lambda\left(\boldsymbol{w}_{k}^{\mathfrak{v}}, \boldsymbol{w}_{k}^{\mathfrak{p}}\right)+\sum_{\mathfrak{e} \in \mathscr{E}_{k}^{*}} \Lambda\left(\boldsymbol{r}_{k}^{\mathfrak{e}}, \boldsymbol{r}_{k}^{\mathfrak{e}}\right) \leq C \Lambda\left(\boldsymbol{w}_{k}, \boldsymbol{w}_{k}\right) .
$$


Expanding the terms and using the orthogonality of the discrete Helmholtz decomposition and the weighted inverse estimate [4, Lemma 4], we obtain

$$
\begin{aligned}
\sum_{\mathfrak{v} \in \mathscr{V}_{k}}\left\|\boldsymbol{w}_{k}^{\mathfrak{v}}\right\|_{\Lambda}^{2}+\sum_{\mathfrak{c} \in \mathscr{E}_{k}^{*}}\left\|\boldsymbol{r}_{k}^{\mathfrak{e}}\right\|_{\Lambda}^{2} & =\sum_{\mathfrak{v} \in \mathscr{V}_{k}}\left\|\operatorname{grad}_{r z} \phi_{k}^{\mathfrak{v}}+\boldsymbol{r}_{k}^{\mathfrak{v}}\right\|_{\Lambda}^{2}+\sum_{\mathfrak{c} \in \mathscr{E}_{k}^{*}}\left\|\boldsymbol{r}_{k}^{\mathfrak{e}}\right\|_{\Lambda}^{2} \\
& =\sum_{\mathfrak{v} \in \mathscr{V}_{k}}\left(\left\|\operatorname{grad}_{r z} \phi_{k}^{\mathfrak{v}}\right\|_{r}^{2}+\left\|\boldsymbol{r}_{k}^{\mathfrak{v}}\right\|_{r}^{2}+\left\|\operatorname{curl}_{r z} \boldsymbol{r}_{k}^{\mathfrak{v}}\right\|_{r}^{2}\right)+\sum_{\mathfrak{e} \in \mathscr{E}_{k}^{*}}\left\|\boldsymbol{r}_{k}^{\mathfrak{e}}\right\|_{\Lambda}^{2} \\
& \leq C \sum_{\mathfrak{v} \in \mathscr{Y}_{k}}\left(h_{k}^{-2}\left\|\phi_{k}^{\mathfrak{v}}\right\|_{r}^{2}+\left(1+h_{k}^{-2}\right)\left\|\boldsymbol{r}_{k}^{\mathfrak{v}}\right\|_{r}^{2}\right)+C \sum_{\mathfrak{e} \in \mathscr{E}_{k}^{* *}} h_{k}^{-2}\left\|\boldsymbol{r}_{k}^{\mathfrak{e}}\right\|_{r}^{2} \\
& \leq C h_{k}^{-2}\left(\left\|\phi_{k}\right\|_{r}^{2}+\left\|\boldsymbol{r}_{k}\right\|_{r}^{2}\right) .
\end{aligned}
$$

By (5.15) and (5.16),

$$
\sum_{\mathfrak{v} \in \mathscr{V}_{k}}\left\|\boldsymbol{w}_{k}^{\mathfrak{v}}\right\|_{\Lambda}^{2}+\sum_{\mathfrak{e} \in \mathscr{E}_{k}^{*}}\left\|\boldsymbol{r}_{k}^{\mathfrak{e}}\right\|_{\Lambda}^{2} \leq C h_{k}^{-2} h_{k-1}^{2}\left\|\boldsymbol{w}_{k}\right\|_{\Lambda}^{2},
$$

which proves (5.18). Thus the condition on the existence of the stable decomposition is verified for the smoothing subspaces of (4.1).

A similar and simpler argument verifies the existence of a stable decomposition for the subspaces of (4.2) as well. We omit the details.

\section{Numerical RESUlts}

In this section, we illustrate our previous theoretical results by numerical examples. We will first report the practically observed convergence rate for the approximate solution of the dual mixed problem of Section 3. This will serve as a test of the sharpness of our theoretical error estimates. We will then report the iteration counts for the multigrid V-cycle applied to $\Lambda_{h} \boldsymbol{x}=f$ for a few choices of the domain and $f$. Note that the $\mathrm{V}$-cycle operator can also be used as a preconditioner for $\Lambda_{h}$ and numerical experiments using it so have already been reported in [17] in the context of solving a div-curl system.

For computer implementation of the mixed method, we need to assemble the matrix representations of the operators $A_{h}: \boldsymbol{W}_{h, \diamond} \mapsto \boldsymbol{W}_{h, \diamond}^{\prime}$ and $B_{h}: \boldsymbol{W}_{h, \diamond} \mapsto S_{h}^{\prime}$ defined by

$$
\begin{aligned}
A_{h} \boldsymbol{u}_{h}\left(\boldsymbol{w}_{h}\right) & =\left(\boldsymbol{u}_{h}, \boldsymbol{w}_{h}\right)_{r} \text { for all } \boldsymbol{u}_{h}, \boldsymbol{w}_{h} \in \boldsymbol{W}_{h, \diamond}, \\
B_{h} \boldsymbol{u}_{h}\left(s_{h}\right) & =-\left(\operatorname{curl}_{r z} \boldsymbol{u}_{h}, s_{h}\right)_{r} \text { for all } \boldsymbol{u}_{h} \in \boldsymbol{W}_{h, \diamond}, s_{h} \in S_{h} .
\end{aligned}
$$

Let $\mathrm{A}$ and B denote the matrix representations of $A_{h}$ and $B_{h}$, respectively, in terms of the standard local bases for $\boldsymbol{W}_{h, \diamond}$ and $S_{h}$ (consisting of the Whitney functions $\Phi_{\mathfrak{e}}$, and the indicator functions of triangles). Then (3.14) can be rewritten as the linear system

$$
\begin{aligned}
A z+B^{t} p & =0, \\
-B z & =f,
\end{aligned}
$$

where $\mathbf{z}$ and $\mathrm{p}$ denote the vectors of coefficients in the basis expansions of $\boldsymbol{z}_{h}$ and $p_{h}$, respectively. The vector $f$ is computed from the right hand side of (3.14) as usual. In practice, we compute $\mathrm{p}$ and $\mathrm{z}$ by solving

$$
\begin{aligned}
& \mathrm{Cp}=\mathrm{f}, \\
& \mathrm{Az}=\mathrm{g},
\end{aligned}
$$


TABLE 6.1. Mixed problem convergence rates

\begin{tabular}{|c|l|l|l|l||l|l|}
\hline level & $\left\|\boldsymbol{z}-\boldsymbol{z}_{h}\right\|_{r}$ & order & $\left\|p-p_{h}\right\|_{r}$ & order & $\left\|\Pi_{h}^{S} p-p_{h}\right\|_{r}$ & order \\
\hline 1 & 0.305150 & & 0.123463 & & 0.044430 & \\
2 & 0.152784 & 0.998 & 0.059647 & 1.05 & 0.011144 & 1.995 \\
3 & 0.076486 & 0.998 & 0.029553 & 1.013 & 0.002791 & 1.997 \\
4 & 0.038263 & 0.999 & 0.014743 & 1.003 & 0.000698 & 1.999 \\
5 & 0.019135 & 1 & 0.007367 & 1.001 & 0.000175 & 2 \\
6 & 0.009568 & 1 & 0.003683 & 1 & 0.000044 & 2 \\
7 & 0.004784 & 1 & 0.001842 & 1 & 0.000011 & 1.999 \\
8 & 0.002392 & 1 & 0.000921 & 1 & 0.000003 & 2 \\
\hline
\end{tabular}

where $C=B A^{-1} B^{t}$ and $g=-B^{t} p$. Both of these systems can be solved via the conjugate gradient method as $\mathrm{C}$ and $\mathrm{A}$ are symmetric and positive definite. Note that when solving the first equation, for each application of $\mathrm{C}$, we use another inner conjugate gradient iteration to obtain the result of multiplication by $\mathrm{A}^{-1}$.

In Table 6.1, we report the $L_{r}^{2}(D)$-norm of the observed errors in the mixed method approximations of $\boldsymbol{z}, p$, and $\Pi_{h}^{S} p$. In this case, $f=-3, p=r^{2}$ and $\boldsymbol{z}=(0,3 r)$. The domain $D$ was chosen to be the unit square. The coarsest mesh is obtained by dividing the unit square into two uniform triangles by connecting the points $(0,0)$ and $(1,1)$. This is mesh level 0 . Higher levels are obtained by successive refinements. Each refinement is performed by connecting the midpoints of each edge, so the mesh size reduces by $1 / 2$, and the finest mesh (level 8 ) is roughly of size $1 / 256$. The order of convergence is computed as $\log _{2}\left(e_{j-1} / e_{j}\right)$, where $e_{j}$ is the computed $L_{r}^{2}(D)$-norm of the error at mesh level $j$.

From the table, we observe that the approximations for $z$ and $p$ converge at first order. This convergence for $\boldsymbol{z}$ is in accordance with Theorem 3.3(2). The convergence for $p$ is also in accordance with the theorem, because by triangle inequality

$$
\left\|p-p_{h}\right\|_{r} \leq\left\|p-\Pi_{h}^{S} p\right\|_{r}+\left\|\Pi_{h}^{S} p-p_{h}\right\|_{r}
$$

and although Theorem 3.3.31) asserts that the last term is $O\left(h^{2}\right)$, the first term on the right hand side, being $O(h)$, dominates. That the last term indeed superconverges at double the order is verified in the last row of the table.

Next, we verify the uniform convergence of the multigrid V-cycle algorithm for $\Lambda_{h} \boldsymbol{x}=f$. We apply the $\mathrm{V}$-cycle algorithm to the three different domains shown in Figure 1. Domain I is convex and its revolution is also convex, while Domain II is convex, but its revolution is nonconvex, and Domain III and its revolution are both nonconvex. The initial mesh (level 1) for domains I and II consists of two congruent right triangles, and for domain III, it consists of four congruent right triangles. In all cases, we obtain the next level mesh by connecting the edge midpoints of all triangles.

Table 6.2 reports the convergence rate when $f=0$. We apply the Gauss-Seidel smoother with the subspace decomposition (4.2). For successive finite element spaces $\boldsymbol{W}_{H, \diamond} \subset \boldsymbol{W}_{h, \diamond}$, the prolongation matrix that we used for implementation is the matrix whose $(i, j)$-th entry is $\int_{e_{i}} \boldsymbol{\phi}_{j} \cdot \boldsymbol{t}_{i}$, where $e_{i}$ denotes the $i$-th edge of mesh $\mathscr{T}_{h}$ that is not on $\Gamma_{1}, \boldsymbol{t}_{i}$ denotes the unit tangent vector of this edge, and $\phi_{j}$ is the $j$-th basis function of $\boldsymbol{W}_{H, \diamond}$. The restriction matrix is the transpose of the prolongation matrix. For each fine level mesh, the initial value $\boldsymbol{x}_{0}$ was 


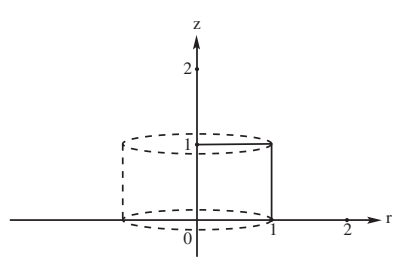

(a) Domain I

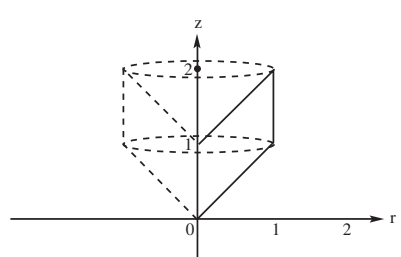

(b) Domain II

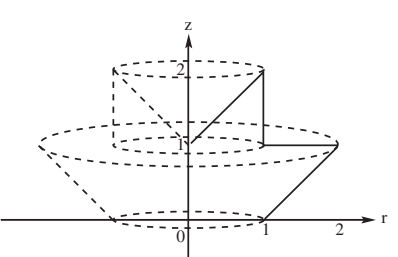

(c) Domain III

Figure 1. Domains

TABLE 6.2. V-cycle convergence rates

\begin{tabular}{|c|c|c|c|}
\hline & \multicolumn{3}{|c|}{ Convergence rate } \\
\hline Level & Domain I & Domain II & Domain III \\
\hline 2 & 0.27 & 0.29 & 0.32 \\
3 & 0.37 & 0.35 & 0.41 \\
4 & 0.43 & 0.41 & 0.40 \\
5 & 0.42 & 0.40 & 0.40 \\
6 & 0.41 & 0.41 & 0.41 \\
7 & 0.41 & 0.41 & 0.41 \\
8 & 0.41 & 0.41 & 0.41 \\
9 & 0.41 & 0.41 & 0.41 \\
\hline
\end{tabular}

chosen randomly using the standard library in $\mathrm{C}++$. The stopping criterion is $\left\|\boldsymbol{x}_{n}\right\|_{r, \text { curl }} /\left\|\boldsymbol{x}_{0}\right\|_{r, \text { curl }}<10^{-7}$, where $\boldsymbol{x}_{n}$ is the result of the $n$-th iteration (which measures the reduction in the error since the exact solution is zero). The convergence rate is computed by taking the average of $\left\|\boldsymbol{x}_{n}\right\|_{r, \text { curl }} /\left\|\boldsymbol{x}_{n-1}\right\|_{r, \text { curl }}$.

As we see from the table, the convergence rate is nearly constant and seems bounded independently of the mesh size. Additionally, although we assumed that the revolution of the two-dimensional domain is convex throughout the paper in order to prove the uniform convergence result, it appears that even when the revolution of the domain is nonconvex, the convergence rate is independent of the mesh size.

\section{Conclusions}

We showed that the multigrid V-cycle converges at a uniform rate with respect to the mesh size when applied to the inner product of $\boldsymbol{H}_{r, \diamond}(\operatorname{curl}, D)$.

Some of the intermediate results we obtained on the way to the multigrid proof are interesting by themselves. For example, we constructed a commuting projector $\Pi_{h}^{W}$ into $\boldsymbol{W}_{h, \diamond}$, whose domain is the entire $\boldsymbol{H}_{r, \diamond}(\operatorname{curl}, D)$ (without any further regularity). We analyzed a dual mixed problem and its discretization and obtained a superconvergence result in weighted norms.

To handle more practical applications, we must extend our results to the time harmonic case, where the additional difficulty of the indefiniteness of the bilinear form will play a role. Furthermore, the design of efficient solvers becomes complicated in practical scattering and radiation problems where we must additionally deal with the truncation of an infinite domain. Finally, since axisymmetric bodies typically have nonaxisymmetric modes excitable by nonaxisymmetric data, we are 
interested in pursuing problems that are "almost" axisymmetric in the sense that solutions can be expanded as a finite series in the $\theta$-variable.

\section{Appendix A. Proof of the exact Sequence Property}

We prove the exactness of the sequence (2.3) under the assumption that $\Gamma_{1}$ is connected and $D$ is simply connected.

The injectivity of $\operatorname{grad}_{r z}: V_{h, \diamond} \mapsto \boldsymbol{W}_{h, \diamond}$ is trivial, so let us proceed to prove the next item in the exact sequence property, namely that $\operatorname{grad}_{r z}\left(V_{h, \diamond}\right)$ equals the null space of $\operatorname{curl}_{r z}: \boldsymbol{W}_{h, \diamond} \mapsto S_{h}$, which is denoted by $\operatorname{ker}\left(\operatorname{curl}_{r z}\right)$. It is obvious that $\operatorname{grad}_{r z}\left(V_{h, \diamond}\right) \subseteq \operatorname{ker}\left(\operatorname{curl}_{r z}\right)$. Since $D$ is simply connected, any $\boldsymbol{w}_{h} \in \boldsymbol{W}_{h, \diamond}$ satisfying $\operatorname{curl}_{r z} \boldsymbol{w}_{h}=0$ coincides with a gradient, say $\operatorname{grad}_{r z} \phi_{h}$, and by comparing the polynomial degrees, $\phi_{h}$ is in $V_{h}$. Moreover, $\phi_{h}$ can be chosen to be in $V_{h, \diamond}$, because $\boldsymbol{t} \cdot \operatorname{grad}_{r z} \phi_{h}=0$ on $\Gamma_{1}$ and because $\Gamma_{1}$ is connected.

Thus, it only remains to prove that $\operatorname{curl}_{r z}: \boldsymbol{W}_{h, \diamond} \mapsto S_{h}$ is surjective. For this we only need the connectedness of $D$ as we see below.

Lemma A.1. The map $\operatorname{curl}_{r z}: \boldsymbol{W}_{h, \diamond} \rightarrow S_{h}$ is surjective.

Proof. The collection of indicator functions $\chi^{K}$, for all mesh elements $K$, spans $S_{h}$. Therefore, to prove the lemma, it suffices to show that there is a $\boldsymbol{u}_{h}^{K}$ in $\boldsymbol{W}_{h, \diamond}$ such that

$$
\operatorname{curl}_{r z} \boldsymbol{u}_{h}^{K}=\chi^{K}
$$

for all mesh elements $K$. To do this we need a commuting projector. We use the projectors $\widehat{\Pi}_{h}^{W}$ and $\widehat{\Pi}_{h}^{S}$ of [17] which we already introduced earlier (although this proof works equally well, mutatis mutandis, with Nédélec's original projector [25]).

To begin, consider all mesh elements $K$ near $\Gamma_{0}$, specifically those in

$$
\mathscr{T}_{h}^{0}=\left\{K \in \mathscr{T}_{h}: \bar{K} \cap \bar{\Gamma}_{0} \text { is nonempty }\right\} .
$$

Let $\alpha_{p}^{K}(\boldsymbol{u})=\left(\int_{K} r^{p} d r d z\right)^{-1} \int_{K} r^{p} \operatorname{curl}_{r z} \boldsymbol{u} d r d z$. Then, for any $K$ in $\mathscr{T}_{h}^{0}$, choose a function $\boldsymbol{u}^{K}$ in $\mathcal{D}(K)^{2}$ with nonzero $\alpha_{1}^{K}\left(\boldsymbol{u}^{K}\right)$. By rescaling this function if necessary, we can assume without loss of generality that $\alpha_{1}^{K}\left(\boldsymbol{u}^{K}\right)=1$. Then consider the interpolant $\boldsymbol{u}_{h}^{K} \equiv \widehat{\Pi}_{h}^{W} \boldsymbol{u}^{K}$. Recalling the definition of $\widehat{\Pi}_{h}^{W}$ in [17, we find that all the degrees of freedom defining $\widehat{\Pi}_{h}^{W}$ applied to $\boldsymbol{u}^{K}$ vanish, except the interior degree of freedom on $K$, namely $\int_{K} r \operatorname{curl}_{r z} \boldsymbol{u}^{K}$. Therefore, by the commutativity property (2.1),

$$
\operatorname{curl}_{r z}\left(\boldsymbol{u}_{h}^{K}\right)=\widehat{\Pi}_{h}^{S} \operatorname{curl}_{r z} \boldsymbol{u}^{K}=\alpha_{1}^{K}\left(\boldsymbol{u}^{K}\right) \chi^{K}=\chi^{K} .
$$

Thus, we have proved A.1 for all $K$ in $\mathscr{T}_{h}^{0}$.

To consider the remaining elements, let $K^{\prime}$ be an element sharing a mesh edge with an element $K$ in $\mathscr{T}_{h}^{0}$. Let $\boldsymbol{u}^{\prime}$ denote an infinitely differentiable vector function supported in $\bar{K} \cup \bar{K}^{\prime}$ such that $\alpha_{0}^{K^{\prime}}\left(\boldsymbol{u}^{\prime}\right) \neq 0$. Then,

$$
\operatorname{curl}_{r z}\left(\widehat{\Pi}_{h}^{W} \boldsymbol{u}^{\prime}\right)=\widehat{\Pi}_{h}^{S}\left(\operatorname{curl}_{r z} \boldsymbol{u}^{\prime}\right)=\alpha_{0}^{K^{\prime}}\left(\boldsymbol{u}^{\prime}\right) \chi^{K^{\prime}}+\alpha_{1}^{K}\left(\boldsymbol{u}^{\prime}\right) \chi^{K} .
$$

Thus, with $\boldsymbol{u}_{h}^{K}$ as set previously, and with $\boldsymbol{u}_{h}^{K^{\prime}}=\left(\widehat{\Pi}_{h}^{W} \boldsymbol{u}^{\prime}-\alpha_{1}^{K}(\boldsymbol{u}) \boldsymbol{u}_{h}^{K}\right) / \alpha_{0}^{K^{\prime}}\left(\boldsymbol{u}^{\prime}\right)$,

$$
\operatorname{curl}_{r z}\left(\boldsymbol{u}_{h}^{K^{\prime}}\right)=\frac{\operatorname{curl}_{r z}\left(\widehat{\Pi}_{h}^{W} \boldsymbol{u}^{\prime}\right)-\alpha_{1}^{K}\left(\boldsymbol{u}^{\prime}\right) \chi^{K}}{\alpha_{0}^{K^{\prime}}\left(\boldsymbol{u}^{\prime}\right)}=\chi^{K^{\prime}}
$$

so that (A.1) is proved for all elements in $\mathscr{T}_{h}^{1}=\left\{K^{1} \in \mathscr{T}_{h} \backslash \mathscr{T}_{h}^{0}: K^{1}\right.$ shares an edge with some $\left.K \in \mathscr{T}_{h}^{0}\right\}$ as well. 
The proof is completed by generalizing to $\mathscr{T}_{h}^{j}=\left\{K^{\prime} \in \mathscr{T}_{h} \backslash \bigcup_{\ell=0}^{j-1} \mathscr{T}_{h}^{\ell}: K^{\prime}\right.$ shares an edge with some $\left.K \in \mathscr{T}_{h}^{j-1}\right\}$, for $j \geq 1$, and formalizing an induction argument.

\section{REFERENCES}

[1] D. N. Arnold, R. S. Falk, And R. Winther, Preconditioning in $H$ (div) and applications, Math. Comp., 66 (1997), pp. 957-984. MR1401938 (97i:65177)

[2] D. N. Arnold, R. S. Falk, and R. Winther, Multigrid in $\mathbf{H}$ (div) and $\mathbf{H}($ curl), Numer. Math., 85 (2000), pp. 197-217. MR.1754719 (2001d:65161)

[3] F. Assous, P. Ciarlet, JR., and S. Labrunie, Theoretical tools to solve the axisymmetric Maxwell equations, Math. Methods Appl. Sci., 25 (2002), pp. 49-78. MR 1874449 (2002j:78008)

[4] Z. Belhachmi, C. Bernardi, and S. Deparis, Weighted Clément operator and application to the finite element discretization of the axisymmetric Stokes problem, Numer. Math., 105 (2006), pp. 217-247. MR2262757 (2008c:65310)

[5] A. Bermúdez, C. Reales, R. Rodríguez, and P. Salgado, Numerical analysis of a finite element method for the axisymmetric eddy current model of an induction furnace, IMA J. Numer. Anal., (To appear).

[6] C. Bernardi, M. Dauge, and Y. Maday, Spectral methods for axisymmetric domains, vol. 3 of Series in Applied Mathematics (Paris), Gauthier-Villars, Éditions Scientifiques et Médicales Elsevier, Paris, 1999. Numerical algorithms and tests due to Mejdi Azaïez. MR.1693480 (2000h:65002)

[7] D. Boffi, P. Fernandes, L. Gastaldi, and I. Perugia, Computational models of electromagnetic resonators: Analysis of edge element approximation, SIAM J. Numer. Anal., 36 (1999), pp. 1264-1290 (electronic). MR1701792 (2000g:65112)

[8] S. BöRm AND R. Hiptmair, Multigrid computation of axisymmetric electromagnetic fields, Adv. Comput. Math., 16 (2002), pp. 331-356. MR.1894928 (2003d:78042)

[9] D. Braess and W. Hackbusch, A new convergence proof for the multigrid method including the $V$-cycle, SIAM J. Numer. Anal., 20 (1983), pp. 967-975. MR714691 (85h:65233)

[10] J. H. Bramble, Multigrid Methods, no. 294 in Pitman research notes in mathematics series, Longman Scientific \& Technical, Harlow, UK, 1993. MR,1247694 (95b:65002)

[11] J. H. Bramble and J. Xu, Some estimates for a weighted $L^{2}$ projection, Math. Comp., 56 (1991), pp. 463-476. MR1066830 (91k:65140)

[12] J. H. Bramble and X. Zhang, The analysis of multigrid methods, in Handbook of Numerical Analysis, Vol. VII, North-Holland, Amsterdam, 2000, pp. 173-415. MR 1804746 (2001m:65183)

[13] F. Brezzi And M. Fortin, Mixed and hybrid finite element methods, vol. 15 of Springer Series in Computational Mathematics, Springer-Verlag, New York, 1991. MR1115205 (92d:65187)

[14] O. Chinellato, The Complex-Symmetric Jacobi-Davidson Algorithm and its Application to the Computation of some Resonance Frequencies of Anisotropic Lossy Axisymmetric Cavities, Dissertation ETH No. 16243, Swiss Federal Institute of Technology, Zürich, 2005.

[15] P. G. Ciarlet, The Finite Element Method for Elliptic Problems, North-Holland Publishing Company, Amsterdam, 1978. MR0520174 (58:25001)

[16] B. Cockburn and J. Gopalakrishnan, Error analysis of variable degree mixed methods for elliptic problems via hybridization, Math. Comp., 74 (2005), pp. 1653-1677 (electronic). MR2164091 (2006e:65215)

[17] D. M. Copeland, J. Gopalakrishnan, and J. E. Pasciak, A mixed method for axisymmetric div-curl systems, Math. Comp., 77 (2008), pp. 1941-1965. MR2429870 (2009e:65171)

[18] D. M. Copeland And J. E. Pasciak, A least-squares method for axisymmetric div-curl systems, Numer. Linear Algebra Appl., 13 (2006), pp. 733-752. MR.2266104 (2008c:78013)

[19] R. FAlk And J. Osborn, Error estimates for mixed methods, RAIRO, 14 (1980), pp. 249-277. MR592753 (82j:65076)

[20] V. Girault AND P.-A. RAviart, Finite element methods for Navier-Stokes equations, Theory and algorithms. vol. 5 of Springer Series in Computational Mathematics, Springer-Verlag, Berlin, 1986. MR851383 (88b:65129) 
[21] J. Gopalakrishnan And J. E. Pasciak, The convergence of V-cycle multigrid algorithms for axisymmetric Laplace and Maxwell equations, Math. Comp., 75 (2006), pp. 1697-1719 (electronic). MR2240631(2007g:65116)

[22] R. Hiptmair, Multigrid method for Maxwell's equations, SIAM J. Numer. Anal., 36 (1999), pp. 204-225 (electronic). MR1654571 (99j:65229)

[23] A. Kufner, O. John, And S. Fučík, Function Spaces, Monographs and textbooks on mechanics of solids and fluids, Noordhoff International Publishing, Leyden, The Netherlands, 1977. MR0482102(58:2189)

[24] P. Lacoste And J. Gay, A new family of finite elements for Maxwell-Fourier's equations, in Mathematical and numerical aspects of wave propagation phenomena (Strasbourg, 1991), SIAM, Philadelphia, PA, 1991, pp. 746-749. MR1106041(92c:65135)

[25] J.-C. NÉDÉLEC, Mixed Finite Elements in $\mathbb{R}^{3}$, Numer. Math., 35 (1980), pp. 315-341. MR.592160 (81k:65125)

[26] J. Nitsche, Ein Kriterium für die Quasi-Optimalität des Ritzschen Verfahrens, Numer. Math., 11 (1968), pp. 346-348. MR0233502 (38:1823)

[27] P.-A. Raviart and J. M. Thomas, Primal hybrid finite element methods for 2nd order elliptic equations, Math. Comp., 31 (1977), pp. 391-413. MR0431752 (55:4747)

Department of Mathematics, Texas A\&M University, College Station, Texas 77843

E-mail address: copeland@math.tamu.edu

Department of Mathematics, University of Florida, Gainesville, Florida 32611-8105

E-mail address: jayg@ufl.edu

Department of Mathematics, University of Florida, Gainesville, Florida 32611-8105

E-mail address: oh@ufl.edu 